\title{
Management of sexual dysfunction in breast cancer survivors: a systematic review

Susan M. Seav ${ }^{1}$, Sally A. Dominick', Boris Stepanyuk', Jessica R. Gorman ${ }^{1,2}$, Diana T. Chingos², Jennifer L. Ehren ${ }^{3}$, Michael L. Krychman ${ }^{4}$ and H. Irene Su${ }^{1 *}$

\begin{abstract}
Female sexual dysfunction occurs frequently in midlife breast cancer survivors (BCS) and encompasses problems with sexual desire, interest, arousal, orgasm and genitopelvic pain. Although common, sexual problems are under-diagnosed and under-treated in BCS. The objective of this review was to assess primary studies that intervene on sexual dysfunction in BCS. In February 2015, PubMed, SCOPUS, CINAHL, COCHRANE and Web of Science databases were systematically searched for randomized controlled clinical trials (RCTs) of vaginal (lubricants, moisturizers, estrogens, dehydroepiandrosterone [DHEA], testosterone, vibrators, dilators), systemic (androgens, anti-depressants, flibanserin, ospemifene), physical therapy (physical activity, pelvic floor training), counseling and educational interventions on sexual function in BCS. Observational studies of vaginal interventions were also included due to the paucity of RCTs. The search yielded 1414 studies, 34 of which met inclusion criteria. Both interventions and outcomes, measured by 31 different sexual function scales, were heterogeneous, and therefore data were not pooled. The review found that regular and prolonged use of vaginal moisturizers was effective in improving vaginal dryness, dyspareunia, and sexual satisfaction. Educational and counseling interventions targeting sexual dysfunction showed consistent improvement in various aspects of sexual health. No consistent improvements in sexual health were observed with physical activity, transdermal testosterone or hot flash interventions. There was a lack of BCS-specific data on vaginal lubricants, vibrators, dilators, pelvic floor therapy, flibanserin or ospemifene. Overall, the quality of evidence for these studies was moderate to very low. Because each of the interventions with BCS data had limited efficacy, clinical trials to test novel interventions are needed to provide evidence-based clinical recommendations and improve sexual function in BCS.
\end{abstract}

Keywords: Breast cancer, Female sexual dysfunction, Systematic review, Cancer survivorship, Vaginal interventions, Sexual health

\section{Introduction}

In the United States, there are more than 2.3 million female cancer survivors who are younger than age 60; $40 \%$ of these women are survivors of breast cancer [1]. Most midlife breast cancer survivors (BCS) undergo surgery, chemotherapy, radiation and/or endocrine therapy for cancer treatment. Receiving a breast cancer diagnosis and undergoing associated treatments including long term endocrine therapy can impair sexual function via a number of mechanisms, including disrupting ovarian

\footnotetext{
* Correspondence: hisu@ucsd.edu

'Department of Reproductive Medicine and Moores Cancer Center,

University of California, San Diego, 3855 Health Sciences Dive \#0901, La Jolla, CA 92093, USA

Full list of author information is available at the end of the article
}

function, body image, intimacy and relationships [2-7]. In turn, impaired sexual function contributes to lower quality of life in survivorship $[8,9]$.

Female sexual dysfunction has been classified into three categories: sexual interest or arousal disorder, orgasmic disorder, and genitopelvic pain or penetration disorder. A women is diagnosed with sexual dysfunction if she experiences persistent symptoms that last at least six months and cause marked distress, as detailed in the Diagnostic and Statistical Manual $5^{\text {th }}$ Edition (DSM-5) [10] (Table 1). A population-based cohort study of recently diagnosed BCS showed $65 \%$ reported that they were sexually active; $52 \%$ of sexually active women described problems with two or more areas of sexual function [11]. At 5 and 10 years after cancer diagnosis, 
Table 1 Female sexual dysfunction classification and diagnostic criteria from the Diagnostic and Statistical Manual of Mental Disorders, Fifth Edition [10]

\begin{tabular}{|c|c|}
\hline Disorder $^{a}$ & Criteria \\
\hline \multirow[t]{7}{*}{ Female sexual interest or arousal disorder } & Absent or significantly decreased sexual interest or arousal as manifested by a lack of or reduction in: \\
\hline & 1. Sexual activity \\
\hline & 2. Sexual or erotic thoughts or fantasies \\
\hline & 3. Initiation of sexual activity and unreceptive to partner's attempts to initiate \\
\hline & 4. Sexual excitement or pleasure during sexual activity in at least $75 \%$ of all sexual encounters \\
\hline & $\begin{array}{l}\text { 5. Sexual interest or arousal in response to any internal or external sexual or erotic cues (written, verbal, or } \\
\text { visual) }\end{array}$ \\
\hline & 6. Genital or non-genital sensations during sexual activity in at least $75 \%$ of all sexual encounters \\
\hline \multirow[t]{3}{*}{ Female orgasmic disorder } & Presence of either of the following in at least $75 \%$ of all sexual activities: \\
\hline & 1. Significant delay in, frequency of, or absence of orgasm \\
\hline & 2. Significantly reduced intensity of orgasmic sensations \\
\hline & 1. Vaginal penetration during intercourse \\
\hline & 2. Significant vulvovaginal or pelvic pain during intercourse or penetration attempts \\
\hline & $\begin{array}{l}\text { 3. Significant fear or anxiety about vulvovaginal or pelvic pain in anticipation of, during, or because of } \\
\text { vaginal penetration }\end{array}$ \\
\hline & 4. Significant tensing or tightening of pelvic floor muscles during attempted vaginal penetration \\
\hline
\end{tabular}

${ }^{\mathrm{a}}$ Symptoms must persist for at least 6 months, cannot be attributed to another nonsexual mental disorder, are not related to or a result of relationship distress or other significant life stressors, and are not a consequence of the effects of a substance, medication, or other medical conditions

prevalence of sexual problems remained significant, 26 and $19 \%$, respectively [12]. These findings that BCS are sexually active and experience sexual dysfunction that persists throughout survivorship have been replicated in multiple cohorts [9, 13-15].

Sexual health is often under-addressed in survivorship care, and only a minority of BCS receives information and education about sexual function from oncology professionals [16]. Among primary care providers at a university-based medical center, $62 \%$ self-reported never or rarely discussing sexual issues with cancer survivors [17]. Providers who perceived having adequate preparedness to evaluate late effects or formal training in survivorship care were more likely to address sexual health considerations. Conversely, lack of knowledge in healthcare providers was a significant barrier to discussions on sex [18]. Moreover, patients may be reluctant or embarrassed to raise sexual concerns with healthcare providers [19]. Only $50 \%$ of BCS thought their providers were knowledgeable about cancer care follow-up and even fewer (41 \%) felt that their providers were equipped to treat their cancer therapy-related symptoms [20]. Hence, disseminating evidence-based information on managing sexual concerns to healthcare providers is a critical aspect of improving sexual health care after breast cancer.

Multiple pharmacologic and behavioral treatments have been tested to improve sexual health after breast cancer. We present a systematic review of primary research on managing sexual dysfunction in breast cancer survivors to generate evidence-based content for improving knowledge on sexual health for BCS and their healthcare providers.

\section{Methods}

\section{Search strategy}

This systematic review was conducted in accordance with PRISMA guidelines [21]. In February 2015, we systematically searched the following databases: PubMed (1966 - February 2015), SCOPUS (1966 - February 2015), CINAHL (Cumulative Index to Nursing and Allied Health Literature) (1981 - February 2015), COCHRANE (all years), and Web of Science (1900 - February 2015). We screened the bibliographies of all included studies for additional references. We sought peer-reviewed articles examining interventions on sexual health among female BCS. We included studies on female breast cancer patients without age restriction and excluded studies on males, nonhumans and other female cancer patients. We included studies on sexual dysfunction, including problems with dyspareunia, sexual pain, vaginismus, vaginal dryness, sexual arousal, desire, and orgasm. For types of interventions, we included vaginal (lubricants, moisturizers, estrogens, dehydroepiandrosterone [DHEA], testosterone, vibrators and dilators), systemic (androgens, anti-depressants, flibanserin, ospemifene), physical therapy (physical activity, pelvic floor training), counseling and educational interventions. We did not include studies on systemic estrogen interventions. For physical therapy, systemic, and counseling 
and educational interventions, we included only randomized controlled clinical trials (RCTs). We retained RCTs and observational studies (cohort and case control studies) on vaginal interventions due to the dearth of RCTs. We excluded qualitative studies and case reports. The final PubMed search strategy is detailed in the Appendix.

\section{Outcome measures}

The primary outcome of this systematic review was sexual function. Measures of sexual function varied widely among studies and are summarized in Table 2.

\section{Data collection}

Three review authors (SS, SD, IS) independently screened the titles and abstracts of all search citations using the inclusion and exclusion criteria. Discrepancies among authors were resolved via consensus. Two of the three review authors (SS, SD, or IS) independently abstracted data on included articles. Data extracted included participants, interventions, sexual health outcome measures, results, and risks of bias (randomization, allocation concealment, blinding, sample size and analysis approach).

Risk of bias for all included studies was assessed independently by two review authors (SD and IS) using the Cochrane risk of bias assessment tool [22]. Discrepancies were resolved by discussion. Studies were evaluated for the following: selection bias (random sequence generation and allocation concealment); performance blinding (blinding of participants and personnel); detection bias (blinding of outcome assessment); attrition bias (incomplete outcome data); reporting bias (selective reporting); and other bias. Each bias criteria was assigned a high, low or unclear risk of bias rating. Additionally, we evaluated the quality of each study using the following GRADE criteria: study limitations (i.e., risk of bias); consistency of effect; imprecision; indirectness and publication bias. RCTs were first classified as high quality, and observational studies were first classified as low quality. All studies were downgraded in quality for any of the following problems: serious limitation to study quality; important inconsistency; uncertainty about directness; imprecise or sparse data; or high probability of reporting bias.

\section{Results}

After searching PubMed $(n=637)$, SCOPUS $(n=665)$, CINAHL $(n=276)$, COCHRANE $(n=220)$ and Web of Science $(\mathrm{n}=186)$ and hand picking $(\mathrm{n}=14), 1984$ articles were retrieved, leaving 1414 articles after removing duplicates. Forty-two full-text articles were accessed, from which 8 were excluded, leaving 34 articles included in this review. The PRISMA flow diagram details study selection results (Fig. 1). No article was excluded because of non-English language.
A total of 31 different sexual health outcome measures were used to assess intervention effects across the $34 \mathrm{pa}-$ pers (Table 2). The Female Sexual Function Index ( $\mathrm{n}=$ 4 studies) and Cancer Rehabilitation Evaluation System ( $\mathrm{n}=3$ studies) were the most commonly used measures. The Vaginal Maturation Index, Vaginal Health Index, and Sexual Activity Questionnaire were each used in 2 separate studies. All other outcome measures were used by single studies. Because of heterogeneity in both intervention and outcome measures, we were unable to pool estimates for a meta-analysis or derive strengths of recommendations based on the GRADE approach.

\section{Vaginal products interventions}

We searched for studies on vaginal lubricants, moisturizers, estrogens, DHEA, testosterone, vibrators and dilators. Eleven studies met inclusion criteria (Tables 3 and 4, Fig 2a). No studies were found on lubricants, DHEA, vibrators and dilators. There were 3 RCTs and 8 single-arm prospective cohorts with no controls. All participants had genitourinary symptoms, experienced $\geq 6$ months of amenorrhea, and completed primary breast cancer treatment. The studies occurred in Australia, Belgium, Germany, Italy, Korea, and the United States. The polycarbophil-based moisturizer Replens ${ }^{\circ}$ was tested in 4 studies involving 133 participants, one in combination with olive oil and pelvic floor muscle relaxation [23-26]; compounded testosterone cream was tested in 2 studies involving 34 participants [27, 28]; $\mathrm{pH}$ balanced lactic acid gel was used in 1 study of 98 participants [29]; and vaginal estrogens were used in 5 studies involving 47 participants [24, 30-33]. Outcomes included patientreported vaginal symptoms, such as dryness, dyspareunia and itching, and vaginal exam-based $\mathrm{pH}$ and cytology.

In women using Replens ${ }^{\text {e, }}$ vaginal dryness decreased in the first week of use [23], with significant additional improvement in dryness, dyspareunia, sexual satisfaction and frequency by 4 and 12 weeks of use [23, 25, 26]. Compared with local vaginal estrogens (estriol or estradiol), Replens ${ }^{\bullet}$ appeared less effective at decreasing vaginal symptoms and improving vaginal histology. However, women who used vaginal estrogens experienced an increase in their serum estradiol levels or decline in gonadotropins, both evidence of systemic absorption [24, 30-33]. At steady state, women on aromatase inhibitors using 25 microgram estradiol tablets twice weekly had low levels of serum estradiol (median $1.3 \mathrm{pg} / \mathrm{mL}$ ) [33]. However, $12 \mathrm{~h}$ after insertion of the tablet, median peak estradiol reached approximately $28 \mathrm{pg} / \mathrm{mL}$ [33]. A pH-balanced gel ( $\mathrm{pH} 4.0)$ decreased vaginal dryness and dyspareunia more than the placebo gel with a higher $\mathrm{pH}$ [29]. Across products, vaginal irritation occurred in $12-50 \%$ of participants, but whether this symptom persisted was not well described. 
Table 2 Sexual function outcome measures

\begin{tabular}{ll}
\hline & Assessment description $^{\text {a }}$ \\
\hline Arizona Sexual Experience Scale (ASEX) $[68,69]$ & 5-item scale measuring sexual drive, arousal,
\end{tabular} vaginal lubrication, orgasm, and satisfaction

Body Image Relationships Scale (BIRS) [70]

Cancer Rehabilitation Evaluation System (CARES) [71]

Changes of Sexual Functioning Questionnaire (CFSQ) [72]

Derogatis Inventory of Sexual Functioning (DISF-SR) [73]

EORTC Quality of Life Questionnaire (QLQ) Breast Cancer Module [74]

Female Sexual Function Index (FSFI) [75]

Functional Assessment of Cancer Therapy (FACT) [76]

Marital Intimacy Questionnaire [77]

Medical Outcomes Study [78]

Menopausal Sexual Interest Questionnaire (MSIQ) [79]

Menopausal Symptom Score [80]

Profile of Female Sexual Function (PFSF) [81]

Psychological Adjustment to Illness Scale (PAIS) [82]

Quality of Marriage Index (QMI) [83]

Study-Specific Scales [50]

Study-Specific Scales [51]

Sexual Activity Questionnaire (SAQ) [84] 11-item Appearance and Sexuality Subscale measuring satisfaction with sexual activity, physical appearance, and body image

Includes 4-item Sexual Interest, 4-item Sexual Function, and 18-item Marital Issues Subscales

Includes 3-item Desire/Interest, and 2-item Frequency/Pleasure Subscales

4-item subscale measuring sexual drive and relationship satisfaction

23-item Sexual Function and Body Image Subscales measuring breast cancer therapy side effects

19-item scale measuring sexual desire, arousal, lubrication, orgasm, satisfaction, and pain

18-item Endocrine Symptoms Subscale (FACT-ES) measuring hormone-related/ menopausa symptoms of breast cancer

8-item scale measuring marital intimacy

4-item Sexual Functioning Subscale measuring sexual dysfunction symptoms

10-item Sexual Satisfaction Subscale measuring desire, responsiveness, and satisfaction in postmenopausal women

Adapted 7-item scale measuring study-specific menopausal symptoms

37-item scale measuring sexual desire, arousal, orgasm, pleasure, concerns, responsiveness, and self-image

46-item clinical interview with Sexual Relationships (PAIS-SR) and Sexual Problems Subscales measuring psychological and social adjustment to illness

6-item scale measuring marital quality

Study-specific scales measuring frequency of sexual desire, intercourse, masturbation, orgasm, initiative for sex, and relationship satisfaction

Study-specific items measuring sexual satisfaction, relationship satisfaction, dyspareunia, and comfort with sexuality

10-item scale with 3 main subscales measuring: Pleasure (SAQ-P; desire, enjoyment, satisfaction), Discomfort (SAQ-D; vaginal dryness, dyspareunia), Habit Subscale (SAQ-H; frequency)

\section{Scoring rubric}

- 6-point Likert scale $(1-6)$; total score 5 - 30

- Higher scores indicate greater sexual dysfunction.

- Score > 19 indicates sexual dysfunction.

- 5-point scale (1 - 5); total score 11 - 55

- Higher scores indicate greater impairment.

- 5-point scale $(0-4)$

- Higher scores indicate greater impairment.

- 5-point Likert scale $(1-5)$; total score 5 - 25

- Higher scores indicate lesser impairment.

- 5- or 9-point scale (depending on item)

- Higher scores indicate less impairment.

- 4-point rating scale; total score 0 - 100 (after linear transformation)

- Higher scores indicate less impairment.

- 6-point Likert scale $(0-5)$; total score 0 - 36

- Higher scores indicate better sexual function.

- Score < 26.5 suggests sexual dysfunction.

- Total score 0 - 72

- Higher scores indicate fewer symptoms.

- 4-point Likert scale $(1-4)$; total score 8 - 32

- Higher scores indicate less impairment.

- 6-point Likert scale (1 - 6)

- Higher scores indicate more symptoms.

- 7-point Likert scale (1-7); total score 10 - 70

- Higher scores indicate less impairment.

- 5-point Likert scale $(0-4)$; total score 0 - 28

- Higher scores indicate more symptoms.

- 5-point Likert scale (1 - 5); total score 0 - 100 (after linear transformation)

- Higher scores indicate less impairment.

- 4-point scale $(0-3)$

- Higher scores indicate poorer adjustment.

- Score $<35$ = good; $35-51=$ fair; $>51=$ poor

- 7-point bipolar scale (1 - 7); total score 6 - 42

- Higher scores indicate better quality of marriage.

- Higher scores indicate less impairment.

- 5- or 6-point Likert scale (depending on item) - Higher scores indicate less impairment.

- 4-point Likert scale $(0-3)$; total score 0 - 24

- Higher scores indicate less impairment. 
Table 2 Sexual function outcome measures (Continued)

\begin{tabular}{|c|c|c|}
\hline \multirow{2}{*}{$\begin{array}{l}\text { Sexual Desire Subscale of Brief Index of Sexual } \\
\text { Function (BISF) [85] }\end{array}$} & \multirow{2}{*}{$\begin{array}{l}\text { Includes 8-item Sexual Desire and 9-item Sexual } \\
\text { Arousal Subscales }\end{array}$} & - 6 - or 7-point Likert scale $(0-5$ or $0-6)$ \\
\hline & & • Higher scores indicate less impairment. \\
\hline \multirow[t]{2}{*}{ Sexual Dysfunction Scale [57] } & \multirow{2}{*}{$\begin{array}{l}\text { 25-item study-specific scale with } 3 \text { subscales } \\
\text { measuring: Behavioral (vaginal dryness, dyspar- } \\
\text { eunia, and frequency), Evaluative (interest, arousal, } \\
\text { and satisfaction), Body image (sense of attractive- } \\
\text { ness, impact of weight change and hair loss) }\end{array}$} & • Total score 0 - 100 \\
\hline & & • Higher scores indicate less impairment. \\
\hline \multirow[t]{2}{*}{$\begin{array}{l}\text { Sexual Function Subscale of Greene } \\
\text { Climacteric Scale (GCS) [86] }\end{array}$} & \multirow[t]{2}{*}{$\begin{array}{l}\text { 21-item scale with } 4 \text { subscales measuring: } \\
\text { Vasomotor Symptoms ( } 2 \text { items), Somatic } \\
\text { Symptoms ( } 7 \text { items; headaches and muscle/joint } \\
\text { pains), Psychological Symptoms ( } 11 \text { items), Sexual } \\
\text { Function ( } 1 \text { item; sexual interest) }\end{array}$} & $\begin{array}{l}\text { - 4-point scale }(0-3) \text {; total score } 0-6 \text { (vaso- } \\
\text { motor), } 0 \text { - } 21 \text { (somatic), } 0-33 \text { (psychological), } \\
0-3 \text { sexual function; combined total score } 0 \text { - } \\
63\end{array}$ \\
\hline & & - Higher scores indicate more symptoms. \\
\hline \multirow[t]{2}{*}{ Sexual Problems Frequency [45] } & \multirow{2}{*}{$\begin{array}{l}\text { Adapted subscale from BIRS measuring frequency } \\
\text { of sexual problems }\end{array}$} & -5-point Likert scale $(0-4)$ \\
\hline & & • Higher scores indicate greater impairment. \\
\hline Sexual Satisfaction Scale (SSS) $[61,87]$ & $\begin{array}{l}\text { Study-specific items measuring relational sexual } \\
\text { satisfaction for male and female partners }\end{array}$ & • Higher scores indicate less impairment. \\
\hline \multirow[t]{2}{*}{ Sexual Satisfaction Scale (SSS) [88] } & \multirow{2}{*}{$\begin{array}{l}\text { 17-item study-specific scale measuring sexual satis- } \\
\text { faction among Korean women }\end{array}$} & - 4-point Likert scale $(1-4)$; total score $17-68$ \\
\hline & & • Higher scores indicate less impairment. \\
\hline \multirow[t]{2}{*}{ Sexual Self Schema Scale [89] } & \multirow{2}{*}{$\begin{array}{l}\text { 50- item, trait-adjectives scale measuring women's } \\
\text { sexual self-perception }\end{array}$} & · 7-point Likert scale $(0-6)$ \\
\hline & & - Higher scores indicate better self-perception. \\
\hline \multirow{2}{*}{$\begin{array}{l}\text { Visual Analog Scale for Vaginal Dryness and } \\
\text { Dyspareunia [29] }\end{array}$} & \multirow{2}{*}{$\begin{array}{l}\text { Linear rating scale measuring vaginal dryness and } \\
\text { pain }\end{array}$} & $\cdot$-10-point scale $(0-10)$ \\
\hline & & • High scores indicate greater impairment. \\
\hline \multirow[t]{2}{*}{ Vaginal Atrophy Symptom [29, 90-92] } & \multirow{2}{*}{$\begin{array}{l}\text { Adapted 3-item scale measuring vaginal dryness, } \\
\text { itching/irritation, dyspareunia }\end{array}$} & - 4-point Likert scale $(0-3)$; total score 0 - 9 \\
\hline & & • Higher scores indicate greater impairment. \\
\hline \multirow[t]{2}{*}{$\begin{array}{l}\text { Vaginal Dryness, Vaginal Itching, Dyspareunia } \\
{[23,32]}\end{array}$} & \multirow[t]{2}{*}{$\begin{array}{l}\text { Study-specific items measuring vaginal dryness, } \\
\text { vaginal itching, and dyspareunia }\end{array}$} & $\begin{array}{l}\text {-5- or 10-point Likert scale (depending on } \\
\text { study) }\end{array}$ \\
\hline & & - Higher scores indicate greater impairment. \\
\hline \multirow[t]{2}{*}{ Vaginal Health Index (VHI) [93, 94] } & \multirow{2}{*}{$\begin{array}{l}\text { 6-parameter gynecological examination rating } \\
\text { appearance of vaginal mucosa }\end{array}$} & - Score of 1 to 5 ; total score 6 - 30 \\
\hline & & - Higher index indicates healthier appearance. \\
\hline \multirow[t]{3}{*}{ Vaginal Maturation Index (VMI) [91, 95] } & \multirow{3}{*}{$\begin{array}{l}\text { Gynecological examination determining vaginal } \\
\text { atrophy }\end{array}$} & · Score of $0-100(\%)$ \\
\hline & & - Higher scores indicate less vaginal atrophy. \\
\hline & & - A score of $<50$ indicates vaginal atrophy. \\
\hline \multirow[t]{2}{*}{ Vaginal Symptoms Score (VSS) $[24,95]$} & \multirow{2}{*}{$\begin{array}{l}\text { Study-specific scale measuring severity of vaginal } \\
\text { atrophy }\end{array}$} & $\cdot$ - 5-point Likert scale $(0-4)$ \\
\hline & & - Higher scores indicate more symptoms. \\
\hline
\end{tabular}

${ }^{a}$ All scales were designed as self-report questionnaires (unless otherwise reported as an examination, interview, or visual analog scale)

Two studies without control participants intervened with vaginal compounded testosterone in BCS on aromatase inhibitors [27, 28]. Compared to baseline measures, 4 weeks of vaginal testosterone improved all domains of the Female Sexual Function Inventory (FSFI) and vaginal atrophy symptoms. One study found $10 \%$ $(n=2)$ of women had detectable serum estradiol levels after testosterone, though both estradiol levels were very low, $<8 \mathrm{pg} / \mathrm{mL}$ [28].

\section{Systemic therapy interventions}

We sought studies using systemic androgens, antidepressants, ospemifene and flibanserin to intervene on sexual function (Tables 5 and 6, Fig 2b). No studies on ospemifene or flibanserin were found. Three randomized, double-blind cross-over trials on androgens and antidepressants were included. All participants completed primary cancer treatment. The studies were conducted in Brazil, Netherlands, and the United States. In the single study on applying daily testosterone cream to the skin for one month, testosterone in postmenopausal cancer survivors did not result in greater sexual desire, pleasure or function than placebo cream [34]. This study accepted all cancer types, with $73 \%$ of the 150 participants on tamoxifen or aromatase inhibitor, suggesting that they are breast cancer survivors. No increases in estradiol were noted while on testosterone cream, consistent with prior studies in women without history of breast cancer [35-40]. Two additional trials involving 115 participants intervened on hot flashes as the primary outcome with venlafaxine, 


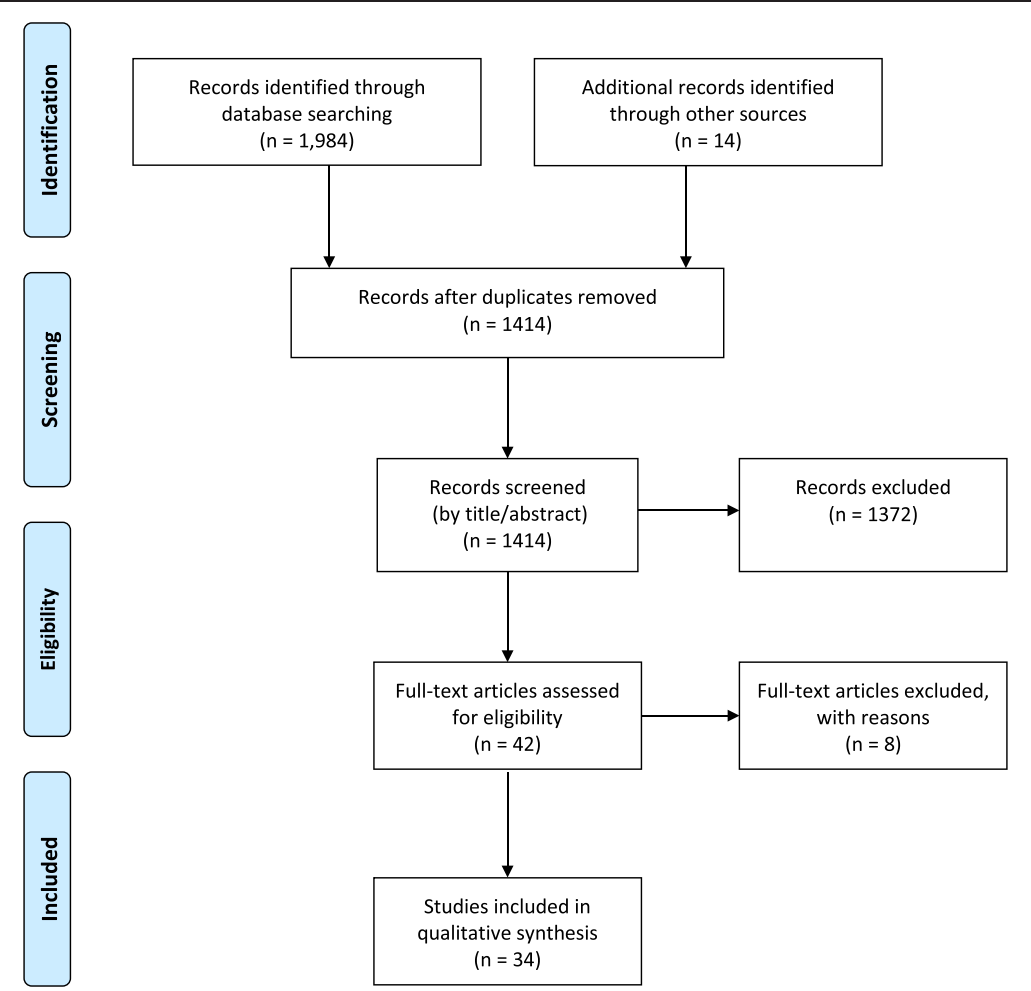

Fig. 1 PRISMA Flow Diagram [21]. Description of articles included throughout the different phases of the systematic review

clonidine or bupropion and examined if sexual function differed by these medications $[41,42]$. There were no differences in sexual function between women treated with venlafaxine compared to clonidine nor with women treated with bupropion versus placebo $[41,42]$.

\section{Physical therapy interventions}

Three RCTs tested physical activity interventions on the primary outcomes of hot flashes, lymphedema, or physical strength and measured sexual health secondarily (Tables 5 and 6, Fig 2c). All participants completed primary breast cancer treatment. There were no studies on pelvic floor physical therapy. Included studies were conducted in the Netherlands, Sweden and United States. A home-based, self-directed exercise program intervened on 422 BCS and did not improve sexual habit, frequency or discomfort as measured by the Sexual Activity Questionnaire [43]. In the two arms with cognitive behavioral therapy, with or without exercise, there was a modest effect on improving sexual health habit at 24 weeks when compared to waitlist controls. Strength training over one year in the second trial of 295 participants was associated with a small improvement in self-perceptions of appearance and sexuality [44]. Finally, a general physical training and coping skills intervention in 199 cancer survivors $(80 \%$ with breast cancer) did not directly address sexual health and did not find change in frequency of sexual problems [45].

\section{Counseling and educational interventions}

Seventeen RCTs delivered counseling and/or educational interventions and measured sexual health outcomes in a total of 2,494 participants (Tables 5 and 6, Fig 2d). Participants were studied at various stages of cancer treatment. Studies were conducted in Australia, Finland, Greece, Korea, Netherlands, United Kingdom, and United States. Nine studies targeted sexual health as the primary outcome [46-54]. There was considerable heterogeneity on intervention and outcome measurements. Twelve studies intervened on the individual, while 5 studies intervened on the couple. The majority delivered in-person interventions, many with additional telephone-support [46, 53, 55-58]. Two recent studies tested web-based interventions [46, 54]. Counseling strategies varied widely, from problem-solving therapy to sexual therapy to cognitive behavioral therapy. Most interventions were delivered by nurses, psychologists, social workers, or peers.

Several findings were consistent. In studies designed specifically to intervene on sexual health, improvements in sexual function were observed in the intervention group compared to controls [46, 48-51], but effect sizes were generally modest and of unclear clinical significance. For example, a 4-month trial tested behavioral and non-estrogen replacement pharmacologic interventions on menopausal symptoms in 76 BCS [48]. The 
Table 3 Summary of studies

\begin{tabular}{|c|c|c|c|c|c|}
\hline \multicolumn{6}{|c|}{ Vaginal products interventions } \\
\hline Reference & Study design & Participants $^{\mathrm{a}}$ & Intervention description & Intervention frequency & Outcome measures \\
\hline Biglia (2010) [24] & $\begin{array}{l}\text { Prospective cohort } \\
\text { study }\end{array}$ & $\begin{array}{l}\text { - Sample size }=31 \\
\text { - Mean age } 54.1 \text { in estrogen groups; } \\
46.1 \text { in polycarbophil-based moisturizer } \\
\text { group }\end{array}$ & 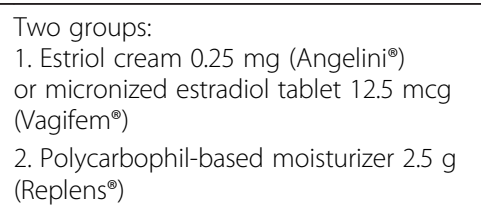 & $\begin{array}{l}\text { 12-week intervention: } \\
\text { • Product twice weekly }\end{array}$ & $\begin{array}{l}\text { 1. Vaginal Symptoms Score } \\
\text { 2. Profile of Female Sexual } \\
\text { Function } \\
\text { 3. Vaginal Health Index }\end{array}$ \\
\hline Dahir (2014) [27] & $\begin{array}{l}\text { Prospective cohort } \\
\text { study }\end{array}$ & $\begin{array}{l}\text { - Sample size }=13 \\
\text { - Mean age } 59.7 \\
\text { - Aromatase inhibitor treatment }\end{array}$ & $\begin{array}{l}\text { One group: } \\
\text { 1. Vaginal compounded testosterone } \\
300 \mathrm{mcg} \text { in } 0.5 \mathrm{~mL}\end{array}$ & $\begin{array}{l}\text { 4-week intervention: } \\
\text { • Daily for } 4 \text { weeks }\end{array}$ & 1. Sexual function (FSFI) \\
\hline Donders (2014) [32] & $\begin{array}{l}\text { Prospective cohort } \\
\text { study }\end{array}$ & $\begin{array}{l}\text { - Sample size }=16 \\
\text { - Mean age } 57.0 \text {, range } 52-63 \\
\text { - Aromatase inhibitor treatment }\end{array}$ & $\begin{array}{l}\text { One group: } \\
\text { 1. Tablet with } 0.03 \mathrm{mg} \text { estriol and } L \text {. } \\
\text { acidophilus (Gynoflor })\end{array}$ & $\begin{array}{l}\text { 12-week intervention: } \\
\text { - Daily for } 4 \text { weeks, then } \\
3 \text { times weekly for } 8 \text { weeks }\end{array}$ & $\begin{array}{l}\text { 1. Serum estradiol, estrone } \\
\text { 2. Serum estriol } \\
\text { 3. Vaginal symptoms } \\
\text { 4. Vaginal } \mathrm{pH} \\
\text { 5. Vaginal maturation } \\
\text { index } \\
\text { 6. Serum FSH } \\
\text { 7. Serum LH }\end{array}$ \\
\hline Gelfand (1994) [25] & $\begin{array}{l}\text { Prospective cohort } \\
\text { study }\end{array}$ & $\begin{array}{l}\text { - Sample size }=25 \\
\text { - Mean age } 60.1 \text {, range } 43-78\end{array}$ & $\begin{array}{l}\text { One group: } \\
\text { 1. Polycarbophil-based moisturizer } 2.5 \mathrm{~g} \\
\text { (Replens }{ }^{\oplus} \text { ) }\end{array}$ & $\begin{array}{l}\text { 12-week intervention: } \\
\text { - Moisturizer three times weekly }\end{array}$ & $\begin{array}{l}\text { 1. Vaginal health index } \\
\text { 2. Vaginal pH } \\
\text { 3. Patient-reported sexual } \\
\text { effects }\end{array}$ \\
\hline Juraskova (2013) [26] & $\begin{array}{l}\text { Prospective cohort } \\
\text { study }\end{array}$ & $\begin{array}{l}\cdot \text { Sample size }=25 \\
\text { - Mean age }=51 \text {, range } 37-66 \\
\text { - In a sexual relationship }\end{array}$ & $\begin{array}{l}\text { One group: } \\
\text { 1. Polycarbophil-based moisturizer } 2.5 \mathrm{~g} \\
(\text { Replens })^{\oplus} \text {; Pelvic floor muscle relaxation; } \\
\text { and Organic olive oil }\end{array}$ & $\begin{array}{l}\text { 26-week intervention: } \\
\text { - Moisturizer three times weekly } \\
\text { - Pelvic floor muscle relaxation twice } \\
\text { daily } \\
\text { - Olive oil use with intercourse }\end{array}$ & $\begin{array}{l}\text { 1. Dyspareunia } \\
\text { 2. Sexual Activity } \\
\text { Questionnaire } \\
\text { 3. Sexual satisfaction (FSFI } \\
\text { subscale) } \\
\text { 4. Endocrine symptoms } \\
\text { (FACT-ES) } \\
\text { 5. Satisfaction and } \\
\text { acceptability }\end{array}$ \\
\hline Kendall (2006) [31] & $\begin{array}{l}\text { Prospective cohort } \\
\text { study }\end{array}$ & $\begin{array}{l}\text { - Sample size }=6 \\
\text { - Mean age }=52 \text {, range } 51-59 \\
\text { - Aromatase inhibitor treatment }\end{array}$ & $\begin{array}{l}\text { One group: } \\
\text { 1. Micronized estradiol } 25 \mathrm{mcg} \\
\text { (Vagifem }^{\oplus} \text { ) }\end{array}$ & $\begin{array}{l}\text { 12-week intervention: } \\
\text { - Daily for } 2 \text { weeks then twice weekly }\end{array}$ & $\begin{array}{l}\text { 1. Atrophic vaginitis } \\
\text { symptoms } \\
\text { 2. Serum estradiol } \\
\text { 3. Serum FSH } \\
\text { 4. Serum LH }\end{array}$ \\
\hline
\end{tabular}


Table 3 Summary of studies (Continued)

\begin{tabular}{|c|c|c|c|c|c|}
\hline Lee (2011) [29] & $\begin{array}{l}\text { Randomized } \\
\text { controlled trial } \\
\text { - Double blind } \\
\text { - Placebo control }\end{array}$ & $\begin{array}{l}\text { - Sample size }=98 \\
\text { - Mean age 45.9, range } 34-53 \text { in } \\
\text { intervention group; } 45.0 \text {, range } \\
37-53 \text { in placebo group } \\
\text { - Pre-menopause status prior to } \\
\text { breast cancer diagnosis }\end{array}$ & $\begin{array}{l}\text { Two groups: } \\
\text { 1. } \mathrm{pH} \text {-balanced lactic acid gel (pH 4.0) } \\
\text { 2. Placebo gel (pH 7.2) }\end{array}$ & $\begin{array}{l}\text { 12-week intervention: } \\
\text { • Gel three times weekly }\end{array}$ & $\begin{array}{l}\text { 1. Dryness with pain } \\
\text { 2. Dyspareunia } \\
\text { 3. Vaginal health index } \\
\text { 4. Vaginal pH } \\
\text { 5. Vaginal maturation } \\
\text { index }\end{array}$ \\
\hline \multirow[t]{4}{*}{ Loprinzi (1997) [23] } & \multirow{4}{*}{$\begin{array}{l}\text { Randomized } \\
\text { controlled trial } \\
\text { - Double blind } \\
\text { - Cross-over }\end{array}$} & - Sample size $=52$ & Two groups: & 9-week intervention: & 1. Product preference \\
\hline & & $\begin{array}{l}\text { - Age } \leq 45=22 \% \\
\text { Age } 46-55=38 \%\end{array}$ & \multirow{3}{*}{$\begin{array}{l}\text { 1. Polycarbophil-based moisturizer } 2.5 \mathrm{~g} \\
\text { (Replens }^{\oplus} \text { ), then } \\
\text { Placebo (Hydroxymethylcellulose, } \\
\text { glycerine-delta lactone, hydrogenated } \\
\text { palm oil glyceride, water) } \\
\text { 2. Placebo, then Replens }{ }^{\oplus}\end{array}$} & \multirow{3}{*}{$\begin{array}{l}\text { - First product: daily } \times 5 \text { days, three } \\
\text { times weekly } \times 23 \text { days } \\
\text { - } 1 \text { week washout } \\
\text { - Second product: daily } \times 5 \text { days, three } \\
\text { times weekly } \times 23 \text { days }\end{array}$} & $\begin{array}{l}\text { 2. Vaginal dryness } \\
\text { 3. Dyspareunia }\end{array}$ \\
\hline & & \multirow[t]{2}{*}{ Age $\geq 56=40 \%$} & & & 4. Itching \\
\hline & & & & & \\
\hline \multirow[t]{4}{*}{ Pfeiler (2011) [30] } & \multirow{4}{*}{$\begin{array}{l}\text { Prospective cohort } \\
\text { study }\end{array}$} & \multirow{4}{*}{$\begin{array}{l}\text { - Sample size }=10 \\
\text { - Mean age } 65, \text { range 50-77 } \\
\text { - Aromatase inhibitor treatment }\end{array}$} & \multirow{4}{*}{$\begin{array}{l}\text { One group: } \\
\text { 1. Estriol } 0.5 \mathrm{mg} \text { vaginal tablet }\end{array}$} & \multirow{4}{*}{$\begin{array}{l}\text { 2-week intervention: } \\
\text { - Daily for } 2 \text { weeks }\end{array}$} & 1. Vaginal dryness \\
\hline & & & & & 3. Serum estradiol \\
\hline & & & & & 4. Serum FSH \\
\hline & & & & & 5. Serum LH \\
\hline \multirow[t]{4}{*}{ Wills (2012) [33] } & \multirow{4}{*}{$\begin{array}{l}\text { Cross-sectional } \\
\text { study }\end{array}$} & \multirow{3}{*}{$\begin{array}{l}\text { - Sample size }=48 \\
\text { - Mean age } 60 \text {, range } 49-67 \text { in } \\
\text { vaginal estrogen groups; } 68 \text {, range } \\
53-79 \text { in control group }\end{array}$} & Three groups: & Ongoing interventions: & \multirow[t]{4}{*}{ 1. Serum estradiol } \\
\hline & & & 1. 25 mcg estradiol tablet $\left(\right.$ Vagifem $\left.^{\oplus}\right)$ & 1. Twice weekly ongoing & \\
\hline & & & 2. Vaginal estrogen ring (Estring ${ }^{\oplus}$ & 2. Every 90 days ongoing & \\
\hline & & - Aromatase inhibitor or SERM treatment & 3. Control: no vaginal estrogen & 3. No vaginal estrogen & \\
\hline \multirow[t]{4}{*}{ Witherby (2011) [28] } & \multirow{4}{*}{$\begin{array}{l}\text { Prospective cohort } \\
\text { study }\end{array}$} & - Sample size $=21$ & Two groups: & 4-week intervention: & 1. Serum estradiol \\
\hline & & $\begin{array}{l}\text { - Mean age } 57 \text {, range } 47-66 \text { in } 150 \mathrm{mcg} \\
\text { group; } 56 \text {, range } 45-69 \text { in } 300 \mathrm{mcg} \text { group }\end{array}$ & $\begin{array}{l}\text { 1. Vaginal compounded testosterone } \\
150 \mathrm{mcg} \text { in } 1 \mathrm{~g} \text { cream }\end{array}$ & - Daily for 4 weeks & $\begin{array}{l}\text { 2. Vaginal atrophy } \\
\text { symptom }\end{array}$ \\
\hline & & \multirow[t]{2}{*}{ - Aromatase inhibitor treatment } & \multirow{2}{*}{$\begin{array}{l}\text { 2. Vaginal compounded testosterone } \\
300 \mathrm{mcg} \text { in } 1 \mathrm{~g} \text { cream }\end{array}$} & & 3. Vaginal pH \\
\hline & & & & & $\begin{array}{l}\text { 4. Vaginal maturation } \\
\text { index }\end{array}$ \\
\hline
\end{tabular}

${ }^{a}$ All studies required history of breast cancer; post menopause or $\geq 6$ months of amenorrhea; genitourinary symptoms; and completion of primary cancer treatment for study participation 
Table 4 Summary of findings

\begin{tabular}{|c|c|c|c|c|c|c|}
\hline \multicolumn{7}{|c|}{ Vaginal products interventions } \\
\hline Reference & Outcomes & Intervention results & Control results & Comparisons & $\begin{array}{l}\text { Quality of } \\
\text { evidence } \\
\text { (GRADE) }\end{array}$ & Comments \\
\hline Biglia (2010) [24] & $\begin{array}{l}\text { 1. Vaginal Symptoms Score } \\
\text { 2. Profile of Female Sexual } \\
\text { Function (PFSF) } \\
\text { 3. Vaginal Health Index (VHI) }\end{array}$ & $\begin{array}{l}\text { Baseline to 4-week score } \\
\text { change (SD) } \\
\text { 1. Replens }{ }^{\oplus:}-6.3 \text { (4.3) } \\
\text { Estrogens: -5.3 (4.7) } \\
\text { 2. Not reported } \\
\text { 3. Replens }{ }^{\oplus}+3.0 \text { (1.6) } \\
\text { Estrogens: +5.9 (3.0) } \\
\text { Baseline to 12-week score } \\
\text { change (SD) } \\
\text { 1. Replens }{ }^{\oplus:}-1.3 \text { (5.5) } \\
\text { Estrogens: -11.6 (5.2) } \\
\text { 2. Replens }{ }^{\oplus:}+2.1 \text { (9.3) } \\
\text { Estrogens: +7.2 (5.4) } \\
\text { 3. Replens }{ }^{\oplus:+2.0 ~(3.4) ~} \\
\text { Estrogens: +8.5 (3.6) }\end{array}$ & No control group & $\begin{array}{l}\text { Between group comparisons } \\
\text { 1. } 4 \text { weeks: } p=0.66 \\
12 \text { weeks: } p=0.01 \\
\text { 2. } 12 \text { weeks: } \\
p=0.19 \\
\text { 3. } 4 \text { weeks: } p=0.05 \\
12 \text { weeks: } \\
p=0.02 \\
\text { Within group comparisons } \\
\text { versus baseline } \\
\text { Replens } \\
\text { 1. } 4 \text { weeks: } p=0.01 \\
12 \text { weeks: } p=0.72 \\
\text { 2. } 12 \text { weeks: } p=0.70 \\
\text { 3. } 4 \text { weeks: } p=0.07 \\
12 \text { weeks: } p=0.42 \\
\text { Estrogens } \\
\text { 1. } 4 \text { weeks: } p<0.01 \\
12 \text { weeks: } p<0.01 \\
\text { 2. } 12 \text { weeks: } p=0.03 \\
\text { 3. } 4 \text { weeks: } p<0.01 \\
12 \text { weeks: } p<0.01\end{array}$ & Very Low & $\begin{array}{l}\text { - Dropout: } 16 \% \\
\text { - Estrogens improved all outcomes } \\
\text { more than Replens }{ }^{\oplus} \text {. } \\
\text { - Replens }{ }^{\oplus} \text { showed no change in } \\
\text { sexual function at } 12 \text { weeks. } \\
\text { - Serum estradiol increased } 1.4-3.1 \\
\text { pg/mL in Estrogens group }(p>0.05) \text {. }\end{array}$ \\
\hline Dahir (2014) [27] & 1. Sexual function (FSFI) & $\begin{array}{l}\text { Mean (SD) } \\
\text { 1. Pre } 8.7(3.8) \\
\text { Post } 18.8(7.1)\end{array}$ & No control & $\begin{array}{l}\text { Within group comparisons } \\
\text { versus baseline } \\
\text { 1. } p<0.001\end{array}$ & Low & $\begin{array}{l}\text { - Dropout: } 8 \% \\
\text { - Significant improvement in all FSFI } \\
\text { domains by post-test. }\end{array}$ \\
\hline Donders (2014) [32] & $\begin{array}{l}\text { 1. Serum estradiol, estrone } \\
\text { 2. Serum estriol } \\
\text { 3. Vaginal symptoms } \\
\text { 4. Vaginal } \mathrm{pH} \\
\text { 5. Vaginal maturation index } \\
\text { 6. Serum FSH } \\
\text { 7. Serum LH }\end{array}$ & $\begin{array}{l}\text { Baseline, 4-week } \\
\text { 1. Only } 1 \text { estradiol level } \\
\text { detectable }(1.2 \mathrm{pg} / \mathrm{mL}) \\
\text { 2. Peak estriol } 104.5 \mathrm{pg} / \mathrm{mL} \text {, } \\
15.8 \mathrm{pg} / \mathrm{mL} \\
\text { 3. Improved dryness, } \\
\text { soreness, dyspareunia } \\
\text { 4. Mean } 6.0,4.4 \\
\text { 5. } 31 \%, 72 \%\end{array}$ & No control & $\begin{array}{l}\text { Within group comparisons } \\
\text { versus baseline } \\
\text { 1. Descriptive only } \\
\text { 2. Descriptive only } \\
\text { 3. } p<0.001 \text { for dryness, } \\
\text { soreness } \\
\text { 4. } p<0.001 \\
\text { 5. } p<0.001 \\
\text { 6. } p=0.03\end{array}$ & Low & $\begin{array}{l}\text { - Dropout: None } \\
\text { - } 1 \text { of } 16 \text { participants with detectable } \\
\text { estradiol level }(1.2 \mathrm{pg} / \mathrm{mL}) \\
\text { at day } 28 \text {. }\end{array}$ \\
\hline
\end{tabular}


Table 4 Summary of findings (Continued)

6. Mean 107.9, 98.9

7. Mean 36.5, 34.0

Gelfand (1994) [25] 1. Vaginal health index

2. Vaginal $\mathrm{pH}$

3. Patient-reported sexual effects

1. Mean score (SD)

Baseline $10.1(0.5)$

1-month $10.8(0.4)$

3-month 19.7 (0.7)

2. Mean $\mathrm{pH}(\mathrm{SD})$

Baseline 6.9 (0.2)

1-month $6.8(0.1)$

3-month $4.9(0.2)$

3. Pain-free intercourse:

Baseline $36 \%$

4-month $69 \%$

Sexual satisfaction

improved:

1-month $0 \%$

3-month $77 \%$

Sexual frequency improved:

1 -month $0 \%$

3-month $42 \%$

Juraskova (2013) [26] 1. Dyspareunia (Visual analog score, 0-10)

2. Sexual Activity

Questionnaire (0-24)

3. Sexual satisfaction FSFI

subscale (0.8-6)

4. Endocrine symptoms

(FACT-ES, 0-72)

5. Satisfaction and

acceptability
Mean (SD)

1. Baseline 7.0 (2.4)

4-week 4.4 (2.4)

26-week 2.7 (2.3)

2. Baseline 7.2 (3.2)

4-week 12.3 (4.3)

26-week 11.6 (4.3)

3. Baseline 2.4 (1.4)

4-week 3.3 (1.8)

26-week (3.5 (1.4)

4. Baseline 51 (9.2)

4-week 51.8 (9.9)

26-week 53.8 (8.7)

5. Intervention helpful:

PFM $92 \%$

7. $p>0.05$

No control

Within group comparisons

versus baseline

1. 1-month

$p>0.05$

$>1$ month

$p<0.001$

2. 1-month

$p>0.05$

$>1$ month

$p<0.001$

3. Descriptive only
Within group comparisons versus baseline

1. $p<0.001$

2. $p<0.001$

3. $p<0.001$

4. $p=0.01$

5. Descriptive only
- Dropout: None

- Vaginal irritation in $12 \%$ of participants.
- Dropout: 36 \%

- Maximum gain in sexual satisfaction and dyspareunia occurred by 12 weeks. 
Table 4 Summary of findings (Continued)

Replens $^{\circledast} 88 \%$

Olive oil $76 \%$

Kendall (2006) [31] 1. Atrophic vaginitis symptoms (yes/no) $\quad 2.5$ of 6 had estradiol

No control

Descriptive data - no comparisons

- Dropout: None

2. Serum estradiol, pmol/L levels $>3 \mathrm{pmol} / \mathrm{L}$

3. Serum FSH, IU/I 3. No significant change

4. Serum LH, IU/I 4. No significant change

Lee (2011) [29] 1. Dryness with pain (Visual Baseline, 12-week mean analog score 0-10) scores (SD)

Baseline, 12-week mean scores Between group comparisons (SD)

2. Dyspareunia (Visual

1. $8.2(0.8)$,

1. $7.9(0.9)$,

1. $p=0.001$

analog score 0-10)

$4.2(1.4)$

$6.5(1.5)$

2. $p=0.04$

3. Vaginal health index

2. $8.2(1.0)$,

2. $8.1(1.0)$,

3. $p=0.002$

4. Vaginal $\mathrm{pH}$

$5.5(1.1)$

$6.1(1.4)$

5. Vaginal maturation index

3. $15.8(3.7), 21.0(3.9)$

3. $14.3(3.7), 17.0(3.9)$

4. $p<0.001$

5. $p<0.001$

4. $6.5(1.1)$,

4. $6.2(1.1)$,

$5.0(0.8)$

$5.7(0.9)$

5. $45.5(3.5), 51.2(3.8)$

5. $46.4(3.7), 47.9$ (2.7)

Loprinzi (1997) [23] 1. Product preference 2. Vaginal dryness (scale 0-4)

$1.41 \%$ prefer Replens ${ }^{\circledast} \%$

1. $24 \%$ prefer Placebo \% score decrease after 4 weeks score decrease after 4 weeks

Between group comparisons

1. $p=0.68$

3. Dyspareunia (scale 0-4)

treatment:

treatment:

2. $p=0.3$

4. Itching (scale 0-4)

3. $60 \%$

$3.41 \%$

3. $p=0.05$

4. Not reported

4. Not reported

4. Not reported

Pfeiler (2011) [30] 1. Vaginal dryness (yes/no) 1.5/6 reported

2. Dyspareunia (yes/no)

improvement

No control

3. Serum estradiol, $\mathrm{pg} / \mathrm{mL}$

2. $3 / 5$ reported

4. Serum FSH, $\mathrm{mU} / \mathrm{mL}$

improvement

5. Serum $\mathrm{LH}, \mathrm{mU} / \mathrm{mL}$

3. All estradiol $<10 \mathrm{pg} / \mathrm{mL}$

after treatment

Mean level pre-, post-

4. $4.75 .7,66.0$

5. $5.32 .4,28.9$

Within group comparisons

versus baseline

Moderate

(n)

- Dropout: $12 \%$

- All outcomes favor intervention.

inal irritation in $50 \%$

participants in first 4 weeks.

1. Descriptive only

2. Descriptive only

3. Descriptive only

4. $p=0.01$

5. $p=0.02$

- Dropout: $27 \%$

- Both groups with improved vaginal dryness by 1 week.

- Vaginal side effects in $42 \%$ participants.

- Dropout: None 
Table 4 Summary of findings (Continued)

\begin{tabular}{|c|c|c|c|c|c|c|}
\hline \multirow[t]{3}{*}{ Wills (2012) [33] } & \multirow[t]{3}{*}{ 1. Serum estradiol } & \multirow{2}{*}{$\begin{array}{l}\text { 1. Median level }(95 \% \mathrm{Cl}) \\
\text { pre-, post- } \\
\text { - Vaginal tablet } 2.9 \mathrm{pmol} / \mathrm{L} \\
(2.9-4.9), 45 \mathrm{pmol} / \mathrm{L}(19-89)\end{array}$} & \multirow[t]{3}{*}{$\begin{array}{l}\text { 1. Mean (range) } 3.72 \\
\mathrm{pmol} / \mathrm{L}(3.0-7.7)\end{array}$} & $\begin{array}{l}\text { Between group comparisons } \\
\text { pre-, post- }\end{array}$ & \multirow[t]{3}{*}{ Low } & \multirow{3}{*}{$\begin{array}{l}\text { - Dropout: None } \\
\text { - Systemic absorption occurs with } \\
\text { intravaginal estrogen therapy (ring } \\
\text { or tablet). }\end{array}$} \\
\hline & & & & $\begin{array}{l}\text { 1. Versus vaginal tablet } p=0.93 \text {, } \\
p<0.001 \text {; }\end{array}$ & & \\
\hline & & $\begin{array}{l}\text { - Vaginal ring } 15.0 \mathrm{pmol} / \mathrm{L} \\
(2.9-19), 15 \mathrm{pmol} / \mathrm{L}(1.9-35)\end{array}$ & & $\begin{array}{l}\text { Versus vaginal ring } \\
p<0.014, p<0.014\end{array}$ & & \\
\hline \multirow[t]{12}{*}{ Witherby (2011) [28] } & 1. Serum estradiol & 1. $\%<5 \mathrm{pg} / \mathrm{mL}$ & \multirow[t]{12}{*}{ No control } & 1. $p=0.91$ & \multirow[t]{12}{*}{ Low } & \multirow{2}{*}{$\begin{array}{l}\text { - Dropout: } 10 \% \\
\text { - Two estradiol levels elevated after } \\
\text { testosterone (both }<8 \mathrm{pg} / \mathrm{mL} \text { ). }\end{array}$} \\
\hline & \multirow{2}{*}{$\begin{array}{l}\text { 2. Vaginal atrophy symptom } \\
\text { (Likert scale, } 0-12 \text { ) }\end{array}$} & Baseline $100 \%$ & & 2. $p<0.001$ & & \\
\hline & & 4-week 90 \% & & 3. $p=0.03$ & & \multirow{10}{*}{$\begin{array}{l}\text { - Adverse effects: hair growth/acne } \\
(n=3) \text {, vaginal irritation }(n=3)\end{array}$} \\
\hline & & 2. Mean (SD) & & 4. $p<0.001$ & & \\
\hline & \multirow{8}{*}{$\begin{array}{l}\text { 4. Vaginal maturation index } \\
\text { (VMI) }\end{array}$} & Baseline 5.9 (1.9) & & & & \\
\hline & & 4 week 2.1 (1.8) & & & & \\
\hline & & 3. Median & & & & \\
\hline & & Baseline 5.5 & & & & \\
\hline & & 4-week 5.0 & & & & \\
\hline & & 4. $\% \mathrm{VMI} \geq 10$ & & & & \\
\hline & & Baseline $20 \%$ & & & & \\
\hline & & 4-week $40 \%$ & & & & \\
\hline
\end{tabular}



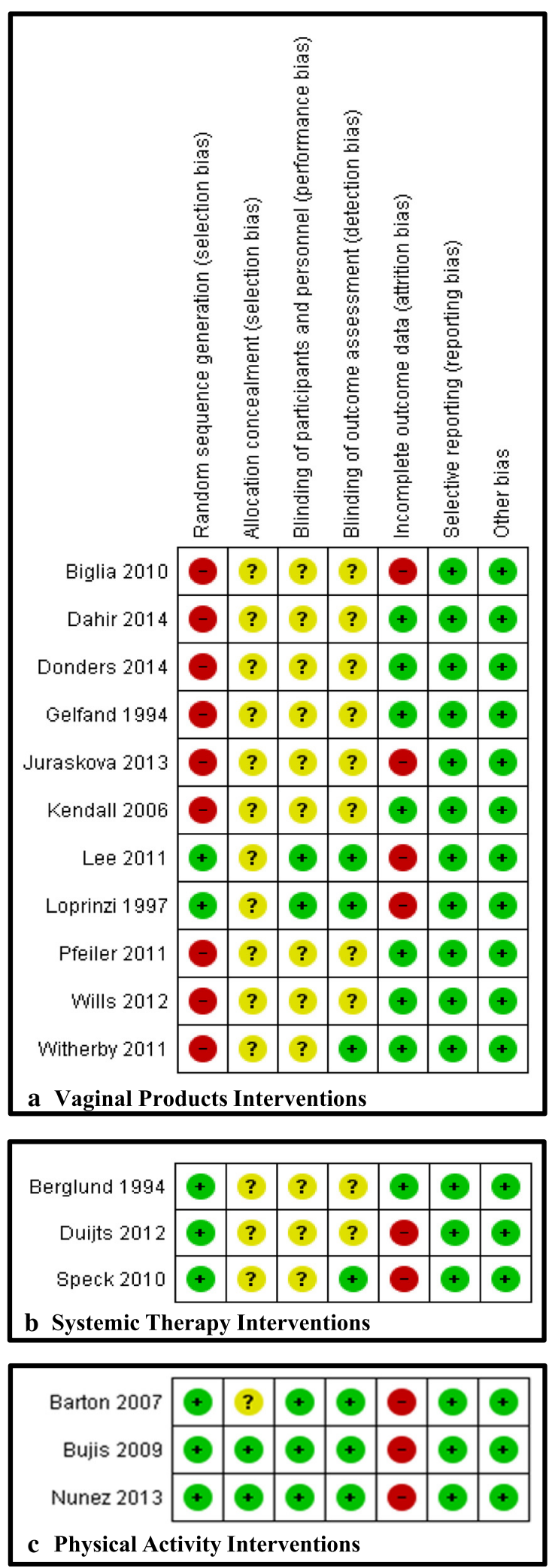

Fig. 2 Risk of Bias Summary. Risk of bias figures detailing the review authors' judgements about each risk of bias item for each included study organized by type of intervention: a Vaginal products interventions, b Systemic therapy interventions, $\mathbf{c}$ Physical activity interventions, $\mathbf{d}$ Counseling and educational interventions

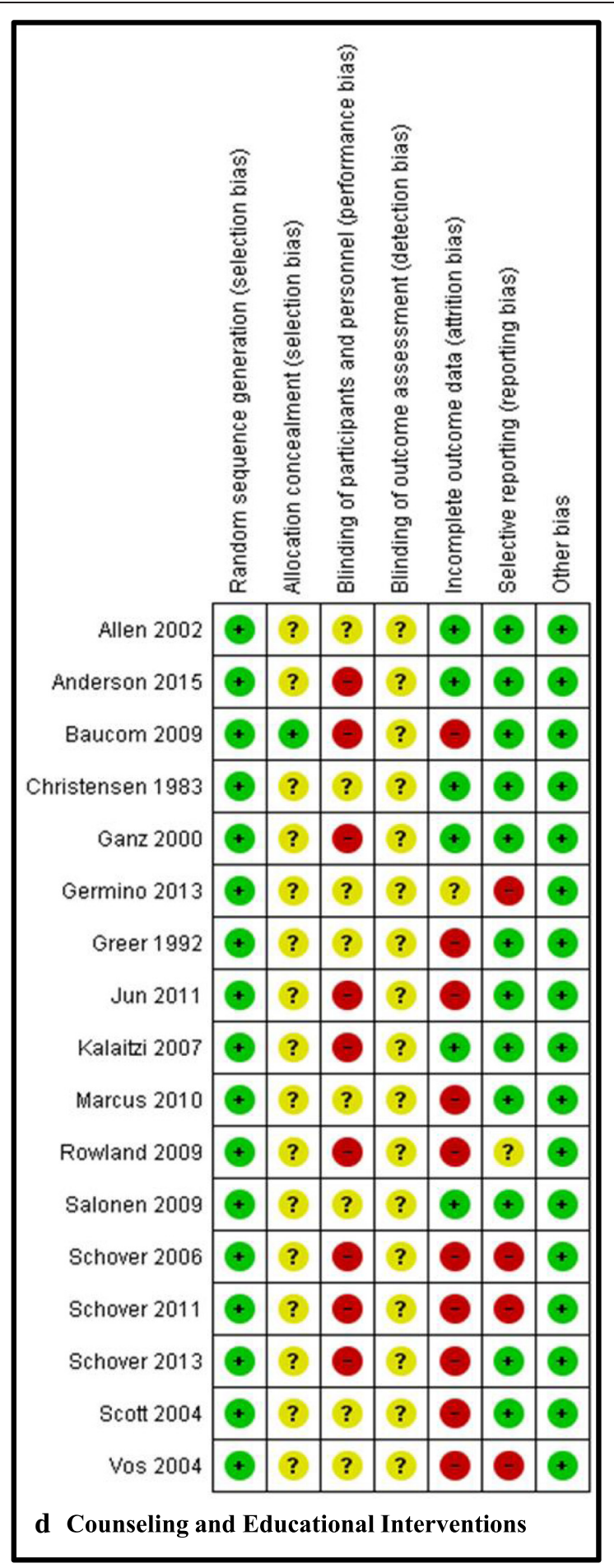

\section{d Counseling and Educational Interventions}


Table 5 Summary of studies

Systemic therapy interventions

\begin{tabular}{|c|c|c|c|c|c|}
\hline Reference & Objective & Participants $^{a}$ & Intervention description & Intervention frequency & Outcome measures \\
\hline Barton (2007) [34] & $\begin{array}{l}\text { To test transdermal testosterone for } \\
\text { increasing sexual desire }\end{array}$ & $\begin{array}{l}\text { - Sample size = } 150 \\
\text { - Mean age } 52.3 \text { (SD 7.9) } \\
\text { - Post-menopause status } \\
\text { - History of any cancer } \\
\text { (73 \% breast cancer) } \\
\text { - Decreased sexual } \\
\text { desire (Changes of } \\
\text { Sexual Functioning } \\
\text { Questionnaire, CSFQ) }\end{array}$ & $\begin{array}{l}\text { Two groups: } \\
\text { 1. Vanicream }+2 \% \text { testosterone }(\mathrm{T}) \text {, } \\
\text { then Vanicream (placebo) } \\
\text { 2. Vanicream (placebo), then Vanicream + } \\
2 \% \text { testosterone cream ( } \mathrm{T} \text { ) } \\
\text { - Testosterone dose } 10.4 \mathrm{mg} \text { daily }\end{array}$ & $\begin{array}{l}\text { 8-week intervention: } \\
\text { - First product: daily x } 4 \\
\text { weeks } \\
\text { - Second product: daily x } 4 \\
\text { weeks }\end{array}$ & $\begin{array}{l}\text { 1. Sexual desire (CSFQ desire } \\
\text { subscale) } \\
\text { 2. Pleasure (CSFQ pleasure } \\
\text { subscale) } \\
\text { 3. Sexual function (CSFQ } \\
\text { total score) } \\
\text { 4. Serum testosterone } \\
\text { 5. Serum estradiol } \\
\text { 6. Serum SHBG } \\
\text { 7. Serum AST }\end{array}$ \\
\hline Buijs (2009) [42] & $\begin{array}{l}\text { To test venlafaxine versus clonidine } \\
\text { on hot flashes }\end{array}$ & $\begin{array}{l}\cdot \text { Sample size }=60 \\
\cdot \text { Median age } 49-51, \\
\text { range } 35-60 \\
\cdot \text { History of breast } \\
\text { cancer } \\
\cdot \geq 14 \text { hot flashes/week }\end{array}$ & $\begin{array}{l}\text { Two groups: } \\
\text { 1. Clonidine }(C) \text { then Venlafaxine }(V) \\
\text { 2. Venlafaxine, then Clonidine or vice } \\
\text { versa } \\
\text { - V: } 75 \mathrm{mg} \text { once daily } \\
\text { - C: } 0.05 \mathrm{mg} \text { twice daily }\end{array}$ & $\begin{array}{l}\text { 18-week intervention: } \\
\text { - One medication daily } \times 8 \\
\text { weeks } \\
\cdot \text {-2-week washout } \\
\text { - Second medication daily } x \\
8 \text { weeks }\end{array}$ & $\begin{array}{l}\text { 1. Sexual function (Sexual } \\
\text { Activity Questionnaire) }\end{array}$ \\
\hline Nunez (2013) [41] & To test bupropion on hot flashes & $\begin{array}{l}\cdot \text { Sample size }=55 \\
\cdot \text { Median age } 49 \text {, range } \\
33-71 \\
\cdot \text { History of breast } \\
\text { cancer } \\
\cdot \geq 7 \text { hot flashes/week }\end{array}$ & $\begin{array}{l}\text { Two groups: } \\
\text { 1. Bupropion (B), then placebo } \\
\text { 2. Placebo then bupropion } \\
\text { - B: titrated to } 300 \mathrm{mg} \text { daily }\end{array}$ & $\begin{array}{l}\text { 10-week intervention: } \\
\cdot \text { One medication daily } \times 3 \\
\text { days, then twice daily for } 25 \\
\text { days } \\
\cdot 1 \text {-week washout } \\
\text { - Second medication daily } x \\
3 \text { days, then twice daily for } \\
25 \text { days }\end{array}$ & $\begin{array}{l}\text { 1. Sexual function (Arizona } \\
\text { Sexual Experience Scale) }\end{array}$ \\
\hline \multicolumn{6}{|c|}{ Physical Activity Interventions } \\
\hline Berglund (1994) [45] & $\begin{array}{l}\text { To test physical training, information } \\
\text { and coping skills training on physical } \\
\text { strength, information need and } \\
\text { mood symptoms }\end{array}$ & $\begin{array}{l}\text { - Sample size }=199 \\
\text { - All cancers ( } 80 \% \\
\text { breast cancer) } \\
\text { - Within } 2 \text { months of } \\
\text { completing primary } \\
\text { cancer treatment }\end{array}$ & $\begin{array}{l}\text { Two groups: } \\
\text { 1. Structured rehabilitation run by oncology } \\
\text { nurse: } \\
\text { Group sessions on physical training, cancer } \\
\text { information, coping training } \\
\text { 2. Controls: Cancer information session with } \\
\text { oncologist/ dietitian }\end{array}$ & $\begin{array}{l}\text { 7-week intervention, 3- } \\
\text { months follow up: } \\
\text { 1. Twice-weekly 2-h sessions } \\
\text { for first } 4 \text { weeks, then one- } \\
\text { weekly } 2 \text {-h session for } 3 \\
\text { weeks } \\
\text { 2. } 0 \text { or } 1 \text { information session }\end{array}$ & $\begin{array}{l}\text { 1. Sexual problems } \\
\text { frequency }\end{array}$ \\
\hline
\end{tabular}


Table 5 Summary of studies (Continued)

\begin{tabular}{|c|c|c|c|c|c|}
\hline Duijts (2012) [43] & To test cognitive behavioral therapy & - Sample size $=422$ & Four groups: & 12-week intervention: & 1. Sexual function (Sexual \\
\hline & $\begin{array}{l}\text { and physical exercise on } \\
\text { menopausal symptoms }\end{array}$ & $\begin{array}{l}\cdot \text { Mean age } 48.2 \text { (SD 5.6) } \\
\cdot \geq 2 \text { menopausal }\end{array}$ & $\begin{array}{l}\text { 1. Cognitive behavioral therapy (CBT): Group } \\
\text { sessions on reducing menopausal symptoms }\end{array}$ & $\begin{array}{l}\text { 1. } 6 \text { weekly groups and } 12^{\text {th }} \\
\text { week refresher }\end{array}$ & $\begin{array}{l}\text { Activity Questionnaire, habit } \\
\text { subscale) }\end{array}$ \\
\hline & & $\begin{array}{l}\text { symptoms over prior } 2 \\
\text { weeks }\end{array}$ & $\begin{array}{l}\text { 2. Physical exercise (PE): Home-based, } \\
\text { self-directed exercise program to achieve } \\
\text { target heart rates, tailored at start by } \\
\text { physiotherapist. }\end{array}$ & $\begin{array}{l}\text { 2. Physiotherapist follow up } \\
\text { in weeks } 4 \text { and } 8\end{array}$ & \\
\hline & & & 3. $C B T / P E$ & & \\
\hline & & & 4. Waitlist control & & \\
\hline Speck (2010) [44] & To test strength training on & - Sample size = 295 & Two groups: & 1-year intervention: & 1. Self-perception of appear- \\
\hline & & • Mean Age 56.5 (SD 9) & 1. Weight-lifting group instruction at & 1. First 13 weeks: twice & $\begin{array}{l}\text { ance and sexuality (Body } \\
\text { Image Relationships Scale, }\end{array}$ \\
\hline & & $\begin{array}{l}\text { - Lymphedema or at risk } \\
\text { for lymphedema }\end{array}$ & exercises, weight-lifting exercises & Remaining of year: twice & $\begin{array}{l}\text { appearance and sexuality } \\
\text { subscale) }\end{array}$ \\
\hline & & & 2. Waitlist control & $\begin{array}{l}\text { weekly unsupervised } \\
\text { exercise; Fitness trainers } \\
\text { called participants if they } \\
\text { missed sessions. }\end{array}$ & \\
\hline & & & & $\begin{array}{l}\text { 2. Maintain usual exercise } \\
\text { level }\end{array}$ & \\
\hline Individual-based Coun & nseling and Educational Interventions & & & & \\
\hline Allen (2002) [55] & To test problem-solving therapy for & - Sample size $=164$ & Two groups: & 16-week intervention: & 1. Sexual health (CARES \\
\hline & & - Mean age 42.3 (SD 5.4) & 1. Problem-solving therapy: Training sessions & 1. 6 biweekly training & Eale) \\
\hline & & $\begin{array}{l}\text { - Initiating } \\
\text { chemotherapy }\end{array}$ & $\begin{array}{l}\text { and manual on problem solving targeting } \\
\text { mid-life breast cancer patients } \\
\text { 2. Controls: No therapy }\end{array}$ & person, 4 on telephone & $\begin{array}{l}\text { 2. Marital relationship } \\
\text { (CARES marital subscale) }\end{array}$ \\
\hline Anderson (2015) [46] & To test a behavioral intervention on & - Sample size $=55$ & Two groups: & 12-week intervention: & 1. Sexual function (sexual \\
\hline & menopausal symptoms & $\begin{array}{l}\cdot \text { Mean age } 49.2 \text { (SD 6.2) } \\
\cdot<12 \text { months from } \\
\text { primary cancer } \\
\text { treatment }\end{array}$ & $\begin{array}{l}\text { 1. Multi-modal tailored program: } \\
\text { Goal setting in consultation with nurse, } \\
\text { follow up calls and emails, written health } \\
\text { education, newsletters and website }\end{array}$ & $\begin{array}{l}\text { 1. Nurse consultation at } \\
\text { weeks } 1,6,12\end{array}$ & $\begin{array}{l}\text { function subscale, Greene } \\
\text { Climacteric Scale) }\end{array}$ \\
\hline & & $\begin{array}{l}\cdot \geq 1 \text { menopausal } \\
\text { symptom as moderate } \\
\text { or severe }\end{array}$ & $\begin{array}{l}\text { 2. Controls: Booklet on breast cancer and } \\
\text { early menopause }\end{array}$ & & \\
\hline Ganz (2000) [48] & To test behavioral and non-estrogen & - Sample size $=76$ & Two groups: & 4-month intervention: & 1. Menopausal Symptom \\
\hline & $\begin{array}{l}\text { replacement pharmaco-logic inter- } \\
\text { ventions on menopausal symptoms }\end{array}$ & $\begin{array}{l}\cdot \text { Mean age } 54.5 \text { (SD 5.9) } \\
\cdot>1 \text { menopausal } \\
\text { symptom as moderate } \\
\text { or severe }\end{array}$ & $\begin{array}{l}\text { 1. Intervention: } \\
\text { Individualized plans of education, counseling, } \\
\text { pharmacologic and/or behavioral } \\
\text { interventions, psychosocial support, referrals } \\
\text { 2. Usual care }\end{array}$ & $\begin{array}{l}\text { 1. Months } 0,2 \text { and } 4 \text { visits } \\
\text { 2. Months } 0 \text { and } 4 \text { visits }\end{array}$ & $\begin{array}{l}\text { Scale Score (hot flash, } \\
\text { vaginal and urinary } \\
\text { subscales of BCPT Symptom } \\
\text { Checklist) } \\
\text { 2. Sexual health (sexual } \\
\text { summary subscale of CARES) }\end{array}$ \\
\hline
\end{tabular}


Table 5 Summary of studies (Continued)

\begin{tabular}{|c|c|c|c|c|c|}
\hline \multirow[t]{3}{*}{ Salonen (2009) [58] } & \multirow{3}{*}{$\begin{array}{l}\text { To test a telephone-based social } \\
\text { support intervention on quality of } \\
\text { life }\end{array}$} & \multirow{3}{*}{$\begin{array}{l}\cdot \text { Sample size }=250 \\
\text { - Mean age } 56-57 \text {, range } \\
24-75 \\
\text { - Newly diagnosed with } \\
\text { breast cancer }\end{array}$} & \multirow{3}{*}{$\begin{array}{l}\text { Two groups: } \\
\text { 1. Telephone support by physiotherapist: } \\
\text { education about illness, at-home exercises, } \\
\text { counseling on stress-related problems, } \\
\text { exploring patient demands and exercises } \\
\text { 2. Control: No telephone support }\end{array}$} & $\begin{array}{l}\text { 1-time intervention, 2-week } \\
\text { follow-up: }\end{array}$ & \multirow[t]{3}{*}{$\begin{array}{l}\text { 1. Sexual functioning } \\
\text { (subscale, EORTC QLQ-BR23) }\end{array}$} \\
\hline & & & & \multirow[t]{2}{*}{$\begin{array}{l}\text { 1. 1-week after breast sur- } \\
\text { gery phone call with therap- } \\
\text { ist (length 3-25 min) }\end{array}$} & \\
\hline & & & & & \\
\hline \multirow[t]{4}{*}{ Schover (2006) [52] } & \multirow{4}{*}{$\begin{array}{l}\text { To test peer counseling on } \\
\text { improving sexual function, } \\
\text { knowledge about reproductive } \\
\text { health, menopausal symptoms and } \\
\text { infertility-related distress }\end{array}$} & - Sample size $=60$ & Two groups: & \multirow{4}{*}{$\begin{array}{l}\text { Immediate counseling } \\
\text { intervention, 3-month follow } \\
\text { up: } \\
\text { 1. } 60-90 \text { min peer counsel- } \\
\text { ing sessions } \times 3\end{array}$} & \multirow{4}{*}{ 1. Sexual Dysfunction (FSFI) } \\
\hline & & & \multirow{3}{*}{$\begin{array}{l}\text { 1. Intervention: In-person peer counselor } \\
\text { sessions reviewing Sisters Peer Intervention } \\
\text { in Reproductive Issues after Treatment } \\
\text { (SPIRIT) } \\
\text { 2. Control: Waitlist control with SPIRIT and } \\
\text { peer counseling at the end of study }\end{array}$} & & \\
\hline & & - African American & & & \\
\hline & & & & & \\
\hline \multirow[t]{3}{*}{ Schover (2011) [53] } & \multirow{3}{*}{$\begin{array}{l}\text { To test peer counseling on } \\
\text { improving sexual function, } \\
\text { knowledge about reproductive } \\
\text { health, menopausal symptoms and } \\
\text { infertility-related distress }\end{array}$} & $\begin{array}{l}\text { - Sample size }=300 \\
\text { - Mean age 54.4 (SD 9.7) }\end{array}$ & \multirow{3}{*}{$\begin{array}{l}\text { Two groups: } \\
\text { 1. Intervention: In-person peer counselor } \\
\text { sessions reviewing Sisters Peer Intervention } \\
\text { in Reproductive Issues after Treatment } \\
\text { (SPIRIT) } \\
\text { 2. Control: Telephone counseling and SPIRIT } \\
\text { workbook }\end{array}$} & $\begin{array}{l}\text { 6-week intervention, 6- } \\
\text { month, 12-month follow up: }\end{array}$ & \multirow[t]{3}{*}{ 1. Sexual Dysfunction (FSFI) } \\
\hline & & $\begin{array}{l}\text { for peer group; } 54.0 \text { (SD } \\
\text { 9.8) for telephone } \\
\text { group }\end{array}$ & & $\begin{array}{l}\text { 1. } 60-90 \text { min peer counsel- } \\
\text { ing sessions } \times 3\end{array}$ & \\
\hline & & - African American & & $\begin{array}{l}\text { 2. } 30 \text { min call to counselor } \\
\text { encouraged } \times 1\end{array}$ & \\
\hline \multirow[t]{4}{*}{ Vos (2004) [60] } & \multirow{4}{*}{$\begin{array}{l}\text { To test a group intervention (group } \\
\text { psycho-therapy or social support) on } \\
\text { psychosocial adjustment }\end{array}$} & \multirow{4}{*}{$\begin{array}{l}\text { - Sample size }=87 \\
\text { - Mean age } 49.2 \text {, range } \\
29-68 \\
\text { - Newly diagnosed with } \\
\text { breast cancer }\end{array}$} & \multirow{4}{*}{$\begin{array}{l}\text { Three groups: } \\
\text { 1. Psychotherapy: Group therapy with } \\
\text { cognitive behavior components } \\
\text { 2. Social support: Group therapy with peer } \\
\text { support } \\
\text { 3. Waitlist control } \\
1 \text { \& } 2 \text { Group interventions discussed fear of } \\
\text { recurrence, coping, body image, sexuality, } \\
\text { intimacy, social support. }\end{array}$} & $\begin{array}{l}\text { 12-week intervention, 3- } \\
\text { month follow up: }\end{array}$ & \multirow[t]{4}{*}{$\begin{array}{l}\text { 1. Sexual function (subscale, } \\
\text { EORTC QLQ-BR32) }\end{array}$} \\
\hline & & & & $\begin{array}{l}\text { 1. Weekly } 2.5 \text { h sessions } x \\
\text { 12; post-treatment } 1 \text { and } 2 \\
\text { month } 2.5 \text { h sessions }\end{array}$ & \\
\hline & & & & 2. Weekly $2.5 \mathrm{~h}$ sessions $\mathrm{x}$ & \\
\hline & & & & $\begin{array}{l}\text { 12; post-treatment } 1 \text { and } 2 \\
\text { month } 2.5 \mathrm{~h} \text { sessions }\end{array}$ & \\
\hline \multicolumn{6}{|c|}{ Couples-based Counseling and Educational Interventions } \\
\hline \multirow[t]{2}{*}{ Baucom (2009) [47] } & \multirow{2}{*}{$\begin{array}{l}\text { To test couple-based relationship } \\
\text { enhancement on relational distress }\end{array}$} & \multirow{2}{*}{$\begin{array}{l}\cdot \text { Sample size }=14 \\
\text { - Median age } 50 \text {, range } \\
30-80 \\
\text { - Married with male } \\
\text { partner }\end{array}$} & Two groups: & \multirow{2}{*}{$\begin{array}{l}\text { 12-week intervention, } 12 \text { - } \\
\text { month follow up: } \\
\text { 1. Biweekly } 75 \text { min sessions } \\
\text { with therapist } \times 6\end{array}$} & \multirow{2}{*}{$\begin{array}{l}\text { 1. Marriage quality (Quality } \\
\text { of Marriage Index) } \\
\text { 2. Sexual function (Derogatis } \\
\text { Inventory of Sexual } \\
\text { Functioning) }\end{array}$} \\
\hline & & & $\begin{array}{l}\text { 1. Relationship enhancement intervention: } \\
\text { Cognitive behavioral therapy on } \\
\text { cancer-related topics } \\
\text { 2. Controls: Community resources list }\end{array}$ & & \\
\hline
\end{tabular}


Table 5 Summary of studies (Continued)

\begin{tabular}{|c|c|c|c|c|c|}
\hline \multirow{3}{*}{$\begin{array}{l}\text { Christensen (1983) } \\
\text { [61] }\end{array}$} & \multirow{3}{*}{$\begin{array}{l}\text { To test a structured couples } \\
\text { treatment program on psychosocial } \\
\text { discomfort }\end{array}$} & - Sample size $=20$ & Two groups: & 6-week intervention: & \multirow{3}{*}{$\begin{array}{l}\text { 1. Sexual satisfaction (Sexual } \\
\text { Satisfaction Scale) }\end{array}$} \\
\hline & & \multirow{2}{*}{$\begin{array}{l}\text { - Mean age } 39.7 \\
\text { - Married with male } \\
\text { partner } \\
\text { - Recent mastectomy }\end{array}$} & $\begin{array}{l}\text { 1. Therapy sessions on communication and } \\
\text { problem solving }\end{array}$ & \multirow[t]{2}{*}{ 1. Weekly sessions $\times 4$} & \\
\hline & & & 2. Controls: No therapy & & \\
\hline \multirow[t]{6}{*}{ Kalaitzi (2007) [50] } & \multirow{6}{*}{$\begin{array}{l}\text { To test combined couples and } \\
\text { sexual therapies on sexual and body } \\
\text { image problems }\end{array}$} & \multirow{5}{*}{$\begin{array}{l}\text { - Sample size }=40 \\
\text { - Mean age } 51.8 \text { for } \\
\text { intervention group, } 53.3 \\
\text { for control group } \\
\text { - Married and sexually } \\
\text { active with male partner }\end{array}$} & Two groups: & 12-week intervention: & \multirow{2}{*}{$\begin{array}{l}\text { 1. Sexual desire frequency } \\
\text { 2. Intercourse frequency }\end{array}$} \\
\hline & & & \multirow{2}{*}{$\begin{array}{l}\text { 1. Intervention: Therapy sessions }-1^{\text {st }} \text { in hospital; } \\
\text { communication training, sensate focus, body } \\
\text { imagery, therapist separation }\end{array}$} & \multirow[t]{3}{*}{ 1. Biweekly sessions $\times 6$} & \\
\hline & & & & & 3. Masturbation frequency \\
\hline & & & \multirow{3}{*}{ 2. Control: no therapy } & & 4. Orgasm frequency \\
\hline & & & & & 5. Initiative for sex \\
\hline & & $\begin{array}{l}\text { - Recent simple } \\
\text { mastectomy }\end{array}$ & & & $\begin{array}{l}\text { 6. Satisfaction with } \\
\text { relationship }\end{array}$ \\
\hline \multirow[t]{7}{*}{ Schover (2013) [54] } & \multirow{7}{*}{$\begin{array}{l}\text { To test an Internet-based interven- } \\
\text { tion, with and without sexual coun- } \\
\text { seling, on sexual function and } \\
\text { satisfaction }\end{array}$} & - Sample size $=72$ & \multirow{4}{*}{$\begin{array}{l}\text { Two groups: } \\
\text { 1. Intervention: In-person counseling to review } \\
\text { website and behavioral homework (both partners) }\end{array}$} & \multirow{7}{*}{$\begin{array}{l}\text { 12-week intervention, 6- } \\
\text { month follow up: } \\
\text { 1. Counseling sessions } \times 3\end{array}$} & \multirow{7}{*}{$\begin{array}{l}\text { 1. Sexual function (FSFI) } \\
\text { 2. Sexual satisfaction } \\
\text { (Menopausal Sexual Interest } \\
\text { Questionnaire) }\end{array}$} \\
\hline & & • Mean age 53 (SD 9) & & & \\
\hline & & - History of breast & & & \\
\hline & & $\begin{array}{l}(80 \%) \text { or gynecologic } \\
\text { cancer }\end{array}$ & & & \\
\hline & & - Sexually active & \multirow{3}{*}{$\begin{array}{l}\text { Both groups: Website on sexual and fertility } \\
\text { consequences of cancer, genital anatomy, } \\
\text { management of vaginal dryness, communication, } \\
\text { dating, treatments for loss of desire, resuming sex } \\
\text { comfortably. }\end{array}$} & & \\
\hline & & $\begin{array}{l}\text { - Sexual dysfunction } \\
\text { (FSFI score }<26.5)\end{array}$ & & & \\
\hline & & $\begin{array}{l}\text { - In a partnered } \\
\text { relationship }\end{array}$ & & & \\
\hline \multirow[t]{4}{*}{ Scott (2004) [96] } & \multirow[t]{4}{*}{$\begin{array}{l}\text { To test a couples-based intervention } \\
\text { on adjustment to cancer }\end{array}$} & \multirow{4}{*}{$\begin{array}{l}\text { - Mean age } 51 \text { (SD 9.8) } \\
\text { - Newly diagnosed } \\
\text { breast (61\%) or } \\
\text { gynecologic cancer } \\
\text { - In a partnered } \\
\text { relationship }\end{array}$} & \multirow{2}{*}{$\begin{array}{l}\text { Three groups: } \\
\text { 1. Couple coping training: Booklet, in-person cou- } \\
\text { ples counseling on coping and support }\end{array}$} & \multirow{4}{*}{$\begin{array}{l}\text { 6-month intervention, } 12- \\
\text { month follow up: } \\
\text { 1. 2-h counseling sessions at } \\
\text { baseline, 1-week, 5-weeks, } 6 \\
\text { months; telephone calls at } 1 \\
\text { and } 3 \text { months } \\
\text { 2. Telephone calls ( }<15 \text { min) } \\
\text { at baseline, } 1 \text { - and } 2 \text {-week } \\
\text { post-surgery, } 6 \text { and } 9 \\
\text { months } \\
\text { 3. 2-h counseling sessions at } \\
\text { baseline, post-surgery, 1- } \\
\text { week, } 6 \text { months; telephone } \\
\text { calls at } 1 \text { and } 3 \text { months }\end{array}$} & $\begin{array}{l}\text { 1. Sexual self schema (Sexual } \\
\text { Self Schema Scale) }\end{array}$ \\
\hline & & & & & \multirow{3}{*}{$\begin{array}{l}\text { 2. Sexual desire (subscale, } \\
\text { Brief Index of Sexual } \\
\text { Function) } \\
\text { 3. Sexual arousal (subscale, } \\
\text { Brief Index of Sexual } \\
\text { Function) } \\
\end{array}$} \\
\hline & & & $\begin{array}{l}\text { 2. Medical information education: Booklet on } \\
\text { cancer and brief telephone calls }\end{array}$ & & \\
\hline & & & $\begin{array}{l}\text { 3. Patient coping training: Booklet and in-person } \\
\text { counseling, telephone calls on coping and } \\
\text { support }\end{array}$ & & \\
\hline
\end{tabular}


Table 6 Summary of findings

Systemic Therapy Interventions

\begin{tabular}{|c|c|c|c|c|c|c|}
\hline Reference & Outcomes & Intervention results & Control results & Comparisons & $\begin{array}{l}\text { Quality of } \\
\text { evidence } \\
\text { (GRADE) }\end{array}$ & Comments \\
\hline \multirow[t]{10}{*}{ Barton (2007) [34] } & \multirow{2}{*}{$\begin{array}{l}\text { All measures normalized to } 100 \text { point } \\
\text { scale: }\end{array}$} & Mean change (95\% Cl): & Mean change $(95 \% \mathrm{Cl})$ : & 1. $p=0.58$ & \multirow[t]{10}{*}{ Moderate } & - Dropout: $12 \%$ \\
\hline & & 1. $5.5(2.2-8.8)$ & 1. $4.4(2.4-6.5)$ & 2. $p=0.11$ & & \multirow{9}{*}{$\begin{array}{l}\text { - Side effects and } \\
\text { quality of life did } \\
\text { not differ by group. }\end{array}$} \\
\hline & 1. Sexual desire (CSFQ desire subscale) & 2. $9.4(7.0-11.2)$ & 2. $4.7(0.4-9.0)$ & 3. $p=0.14$ & & \\
\hline & 2. Pleasure (CSFQ pleasure subscale) & 3. $5.7(4.1-10.6)$ & 3. $3.4(2.1-6.8)$ & \multirow{2}{*}{$\begin{array}{l}\text { 4. } p<0.001 \text { for } \\
\text { both measures }\end{array}$} & & \\
\hline & 3. Sexual function (CSFQ total score) & 4. Total $92.8(74.9-110.7)$ & 4. Total $1.2(-1.8-4.3)$ & & & \\
\hline & 4. Serum testosterone, $\mathrm{ng} / \mathrm{dL}$ & Free 1.6 & Free $0.18(-0.1-0.5)$ & 5. $p=0.82$ & & \\
\hline & 5. Serum estradiol, pg/mL & $(1.2-2.0)$ & 5. $0.5(-5.2-6.1)$ & 6. $p=0.11$ & & \\
\hline & 6. Serum SHBG, nmol/L & 5. $-0.3(-2.9-2.4)$ & 6. $-0.3(-3.0-2.3)$ & \multirow[t]{2}{*}{ 7. $p=0.93$} & & \\
\hline & \multirow[t]{2}{*}{ 7. Serum AST, U/L } & 6. $-3.1(-5.1--1.0)$ & 7. $0.2(-1.1-1.5)$ & & & \\
\hline & & 7. $-0.23(-1.3-0.8)$ & & & & \\
\hline \multirow[t]{2}{*}{ Buijs (2009) [42] } & \multirow{2}{*}{$\begin{array}{l}\text { 1. Sexual function (Sexual Activity } \\
\text { Questionnaire) }\end{array}$} & 1. Venlafaxine: No change & 1. No control & \multirow[t]{2}{*}{ 1. Not reported } & \multirow[t]{2}{*}{ Moderate } & \multirow[t]{2}{*}{ • Dropout: $33 \%$} \\
\hline & & Clonidine: No change & & & & \\
\hline \multirow[t]{2}{*}{ Nunez (2013) [41] } & \multirow{2}{*}{$\begin{array}{l}\text { 1. Sexual function (Arizona Sexual } \\
\text { Experience Scale) }\end{array}$} & Pre- to post- difference (SD): & Pre- to post- difference (SD): & \multirow[t]{2}{*}{ 1. $p=0.5$} & \multirow[t]{2}{*}{ Moderate } & \multirow[t]{2}{*}{ • Dropout: $11 \%$} \\
\hline & & 1. $1.4(3.8)$ & 1. $0.6(3.4)$ & & & \\
\hline \multicolumn{7}{|c|}{ Physical activity interventions } \\
\hline \multirow[t]{2}{*}{ Berglund (1994) [45] } & \multirow[t]{2}{*}{ 1. Sexual problems frequency (Scale 0-4) } & Pre, post, 3-month mean scores (SD): & Pre, post, 3-month mean scores (SD): & \multirow{2}{*}{$\begin{array}{l}\text { 1. Not } \\
\text { significant }\end{array}$} & \multirow[t]{2}{*}{ High } & \multirow[t]{2}{*}{ - Dropout: $8 \%$} \\
\hline & & 1. $0.7(1.2), 0.6(1.0), 0.5(1.0)$ & 1. $0.6(1.0), 0.5(0.9), 0.4(0.7)$ & & & \\
\hline \multirow[t]{6}{*}{ Duijts (2012) [43] } & \multirow{6}{*}{$\begin{array}{l}\text { 1. Sexual function (Sexual Activity } \\
\text { Questionnaire, habit subscale) }\end{array}$} & Baseline, 12-week mean scores (SD): & Baseline, 12-week mean scores (SD): & \multirow{4}{*}{$\begin{array}{l}\text { Effect size } \\
\text { (intervention to } \\
\text { waitlist control } \\
\text { at } 12 \text { weeks): } \\
\text { 1. CBT: } 0.31, p \\
=0.13\end{array}$} & Moderate & • Dropout: $17 \%$ \\
\hline & & 1. CBT: $0.3(0.8), 0.5(0.8)$ & 1. $0.6(0.8), 0.6(0.8)$ & & & - Significant under- \\
\hline & & PE: $0.6(0.8), 0.6(0.8)$ & & & & \\
\hline & & CBT/PE: $0.4(0.8), 0.5$ (0.8) & & & & $58 \%$ CBl; $64 \%$ PE; \\
\hline & & & & $\begin{array}{l}\text { PE: } 0.01 \\
p=0.97\end{array}$ & & Th \\
\hline & & & & $\begin{array}{l}\text { CBT/PE: } 0.15 \\
p=0.44\end{array}$ & & \\
\hline Speck (2010) [44] & 1. Self-perception of appearance and & Mean \% change (SD): & Mean \% change (SD): & 1. $p=0.004$ & Moderate & • Dropout: $21 \%$ \\
\hline & $\begin{array}{l}\text { sexuality (Body Image Relationships Scale } \\
\text { appearance and sexuality subscale) }\end{array}$ & 1. $7.3(16.6)$ & 1. $-0.7(18.1)$ & & & \\
\hline
\end{tabular}


Table 6 Summary of findings (Continued)

Individual-based counseling and educational interventions

2. Marital relationship (CARES marital
subscale)

Anderson (2015) [46] 1. Sexual function (sexual function subscale, Greene Climacteric Scale)

Baseline, 4-month mean scores (SD):

1. $2.2(1.1), 2.1(0.9)$

2. $1.8(0.8), 1.7(0.7)$

Baseline, 12-week mean score (SD) and Baseline, 12-week mean score (SD) and 1. $p=0.05$;

effect size Cohen's $d$ :

$$
\begin{aligned}
& \text { 1. } 2.0(1.0), 1.3(1.0) \\
& d=0.65
\end{aligned}
$$

Ganz (2000) [48] 1. Menopausal Symptom Scale Score (hot flash, vaginal and urinary subscales of BCPT Symptom Checklist)

Mean change score $(95 \% \mathrm{Cl})$ :

1. 0.57

2. Sexual health (sexual summary

$(0.40-0.74)$ subscale of (ARES)

2. 0.46

$(0.30-0.62)$ Study - Sexual Functioning)

Mean score (SD) at baseline, 4-6 months, 8-10 months:

1. $2.1(1.0), 2.0(1.0), 2.0(1.1)$

Mean difference (SD) from baseline to 1. Sexual relationships (subscale of Psychological Adjustment to Illness Scale) 8-weeks, to 4-months:

1. $0.7(7.2),-1.3(7.7)$

Jun (2011) [49]

1. Marital intimacy (Martial Intimacy Questionnaire)

Change in mean (SD) scores:

2. Sexual interest (subscale, CARES)

1. $+2.0(5.0)$

2. $-0.2(0.6)$

3. $-0.1(1.0)$

3. Sexual dysfunction (subscale,

4. Sexual satisfaction (Sexual Satisfaction Scale)

4. $+5.3(9.0)$

1. Sexual function, (behavioral, evaluative and body image subscales of Sexua

Baseline, 12- and 18-month mean scores Baseline, 12- and 18-month mean scores (approximated from figure):

Dysfunction Scale)

1. $47,40,40$
Baseline, 4-month mean scores (SD):

1. $p>0.05$

2. $p>0.05$

$$
\text { effect size Cohen's } d \text { : }
$$

3. $1.6(1.1), 1.4(1.0)$

$d=0.18$

Mean change

score $(95 \% \mathrm{Cl})$ :

1. 0.09

$(-0.04-0.21)$

2. 0.11

$(-0.16-0.38)$

Mean score (SD) at baseline, 4-6 months, 8-10 months:

1. $2.3(1.1), 2.3(1.1), 2.2(1.1)$ (approximated from graph):

1. $45,43,43$
High

- Dropout: $9 \%$

Moderate • Dropout: $9 \%$

Cohen's $d_{2}$ of

post-

intervention

1. $p<0.01$

2. $p=0.03$

Moderate - Dropout: 5 \%

- Both groups used

educational

materials.

- Intervention

group more likely

to receive

pharmaco-logic

and behavioral

interventions.

1. $p=0.03$ at 4- Moderate - Dropout: None 6 months fol-

low up

- Single time point statistically differen without adjustment for baseline differences.

Mean difference (SD) from baseline to $\quad$ 1. $p=0.53$ at 8- Moderate • Dropout: $21 \%$ 8-weeks, to 4- months:

week, $p=0.47$

at 4-months

Change in mean (SD) scores:

1. $p=0.29 \quad$ Low $\quad$ Dropout: $25 \%$

2. $p=0.45$

3. $p=0.53$

4. $p<0.001$

3. $+0.1(1.1)$

4. $-3.4(5.8)$

Comparison by Moderate · Dropout: $20 \%$

intervention

group:

1. $p=0.03$ at

12-month,

$p=0.04$ at 18

month 
Table 6 Summary of findings (Continued)

\begin{tabular}{|c|c|c|c|c|c|c|}
\hline \multirow[t]{7}{*}{ Rowland (2009) [51] } & Likert scales: & Mean change in score (SD): & Mean change in score (SD): & Per-protocol & Low to & • Dropout: $56 \%$ \\
\hline & \multirow{2}{*}{$\begin{array}{l}\text { 1. Satisfaction with variety of sexual } \\
\text { activities }\end{array}$} & & & comparisons: & & \multirow{6}{*}{$\begin{array}{l}\text { - Intervention } \\
\text { group: } 89 / 284 \\
\text { ( } 29 \% \text { ) agreed to } \\
\text { participate; } 72 / 284 \\
\text { ( } 25 \%) \text { attended } \geq 1 \\
\text { session. }\end{array}$} \\
\hline & & 1. $0.1(1.2)$ & 1. $-0.03(1.0)$ & 1. $p=0.23$ & & \\
\hline & 2. Relationship satisfaction & 2. $0(1.5)$ & 2. $-0.3(1.0)$ & 2. $p=0.02$ & & \\
\hline & 3. Dyspareunia & 3. $0.7(1.5)$ & 3. $-0.1(1.7)$ & 3. $p=0.09$ & & \\
\hline & 4. Pain interferes with pleasure & 4. $0.3(1.4)$ & 4. $0(1.1)$ & 4. $p=0.29$ & & \\
\hline & 5. Improved comfort with sexuality & 5. Not reported & 5. Not reported & 5. $p=0.03$ & & \\
\hline \multirow[t]{2}{*}{ Salonen (2009) [58] } & \multirow{2}{*}{$\begin{array}{l}\text { 1. Sexual functioning (subscale 0-100, } \\
\text { EORTC QLQ-BR23) }\end{array}$} & Mean score (SD): & Mean score (SD): & \multirow[t]{2}{*}{ 1. $p=0.2$} & \multirow[t]{2}{*}{ High } & \multirow[t]{2}{*}{ • Dropout: $9 \%$} \\
\hline & & 1. $29(26)$ & 1. $24(22)$ & & & \\
\hline Schover (2006) [52] & 1. Sexual Dysfunction (FSFI) & 1. Not reported & 1. Not reported & 1. No difference & Very low & • Dropout: $20 \%$ \\
\hline Schover (2011) [53] & 1. Sexual Dysfunction (FSFI) & 1. Not reported & 1. Not reported & 1. No difference & Very low & • Dropout: $38 \%$ \\
\hline Vos (2004) [60] & 1. Sexual function (subscale, QLQ-BR32) & 1. Not reported & 1. Not reported & $\begin{array}{l}\text { 1. Regression } \\
\text { coefficient } \\
\text { comparing } \\
\text { intervention to } \\
\text { control: }-0.17 \\
(p>0.05)\end{array}$ & Low & • Dropout: $21 \%$ \\
\hline \multicolumn{7}{|c|}{ Couple-based counseling and educational interventions } \\
\hline \multirow[t]{11}{*}{ Baucom (2009) [47] } & $\begin{array}{l}\text { 1. Marriage quality (Quality of Marriage } \\
\text { Index) }\end{array}$ & $\begin{array}{l}\text { Baseline, 12-week, 12-month mean } \\
\text { score (SD): }\end{array}$ & $\begin{array}{l}\text { Baseline, 12-week, 12-month mean } \\
\text { score (SD): }\end{array}$ & \multirow{3}{*}{$\begin{array}{l}\text { Effect size of } \\
\text { treatment to } \\
\text { controls: } \\
\text { Baseline to 12- } \\
\text { weeks } \\
\text { 1. Female } 0.48\end{array}$} & \multirow[t]{11}{*}{ Low } & \multirow[t]{11}{*}{ • Dropout: $14 \%$} \\
\hline & \multirow[t]{10}{*}{$\begin{array}{l}\text { 2. Sexual function (Derogatis Inventory } \\
\text { of Sexual Functioning) }\end{array}$} & $\begin{array}{l}\text { 1. Female: } 34.0 \text { (13.6), } 39.3 \text { (4.7), } 39.7 \\
\text { (3.5) }\end{array}$ & 1. Female: 40.8 (6.0), $42.2(4.1), 40.2(5.1)$ & & & \\
\hline & & Male: 39.3 (6.6), 39.6 (5.9), 39.6 (5.2) & Male: $42.5(3.0), 3 / .5$ (13.6), $41.0(6.2)$ & & & \\
\hline & & 2. Female: 11.5 (5.0), 12.7 (4.1), 13.0 (3.1) & 2. Female: 10.3 (4.8), 9.8 (5.8), 9.8 (5.9) & Male 0.64 & & \\
\hline & & \multirow[t]{7}{*}{ Male: $12.4(1.5), 13.9(2.8), 13.2(2.4)$} & \multirow{7}{*}{ Male: 12.0 (3.9), 12.3 (2.8), $9.4(4.5)$} & 2. Female 0.34 & & \\
\hline & & & & Male 0.38 & & \\
\hline & & & & $\begin{array}{l}\text { Baseline to } 12- \\
\text { months }\end{array}$ & & \\
\hline & & & & 1. Female 0.77 & & \\
\hline & & & & Male 0.34 & & \\
\hline & & & & 2. Female 0.42 & & \\
\hline & & & & Male 1.04 & & \\
\hline \multirow[t]{2}{*}{ Christensen (1983) [61] } & \multirow{2}{*}{$\begin{array}{l}\text { 1. Sexual satisfaction (Sexual Satisfaction } \\
\text { Scale) }\end{array}$} & Post-test mean score (SD): & Post-test mean score (SD): & \multirow{2}{*}{$\begin{array}{l}\text { 1. } p<0.05 \text { for } \\
\text { both partners }\end{array}$} & \multirow[t]{2}{*}{ High } & \multirow[t]{2}{*}{ - Dropout: None } \\
\hline & & 1. Female partner: 80.4 (31.5) & 1. Female partner: 69.0 (20.2) & & & \\
\hline
\end{tabular}


Table 6 Summary of findings (Continued)

\begin{tabular}{|c|c|c|c|c|c|c|}
\hline & & Male partner: 81.3 (28.7) & Male partner: 67.3 (28.6) & & & \\
\hline \multirow[t]{8}{*}{ Kalaitzi (2007) [50] } & 1. Sexual desire frequency & \multirow{2}{*}{$\begin{array}{l}\text { Baseline, 12-week mean scores (95 \% } \\
\text { Cl): }\end{array}$} & \multirow{2}{*}{$\begin{array}{l}\text { Baseline, 12-week mean scores (95\% } \\
\text { (I): }\end{array}$} & \multirow{2}{*}{$\begin{array}{l}\text { 1. } p=0.73 \\
\text { 2. } p=0.14\end{array}$} & & \multirow[t]{8}{*}{ - Dropout: None } \\
\hline & 2. Intercourse frequency & & & & & \\
\hline & 3. Masturbation frequency & \multirow{2}{*}{$\begin{array}{l}\text { 1. } 2.9(2.3-3.4), 2.8(2.3-3.2) \\
\text { 2. } 3.2(2.7-3.6), 2.9(2.5-3.3)\end{array}$} & 1. $3.0(2.6-3.4), 2.7(2.2-3.1)$ & 3. $p=0.32$ & & \\
\hline & 4. Orgasm frequency & & \multirow{2}{*}{ 3. $1.9(1.6-2.2), 1.9(1.5-2.2)$} & 4. $p=0.03$ & & \\
\hline & 5. Initiative for sex & & & 5. $p<0.001$ & & \\
\hline & \multirow[t]{3}{*}{ 6. Satisfaction with relationship } & 4. $3.3(2.8-3.8), 3.7(3.4-4.0)$ & 4. $3.6(3.1-4.0), 3.1(2.6-3.6)$ & \multirow[t]{3}{*}{ 6. $p=0.01$} & & \\
\hline & & 5. $2.2(1.8-2.6), 2.7(2.3-3.0)$ & 5. $2.6(2.2-3.0), 1.8(1.4-2.2)$ & & & \\
\hline & & 6. $3.8(3.3-4.2), 4.5(4.2-4.7)$ & 6. $3.3(2.9-3.7), 3.7(3.2-4.1)$ & & & \\
\hline \multirow[t]{4}{*}{ Schover (2013) [54] } & 1. Sexual function (FSFI) & \multirow{3}{*}{$\begin{array}{l}\text { Linear mixed model coefficients, post- } \\
\text { treatment versus baseline: } \\
\text { 1. } 7.4\end{array}$} & \multirow{3}{*}{$\begin{array}{l}\text { Linear mixed model coefficients, post- } \\
\text { treatment versus baseline: } \\
\text { 1. } 2.8\end{array}$} & \multirow{3}{*}{$\begin{array}{l}\text { 1. } p=0.024 \\
\text { 2. } p=0.01\end{array}$} & \multirow[t]{4}{*}{ Low } & \multirow[t]{4}{*}{ - Dropout: $36 \%$} \\
\hline & \multirow{3}{*}{$\begin{array}{l}\text { 2. Sexual satisfaction (Menopausal Sexual } \\
\text { Interest Questionnaire) }\end{array}$} & & & & & \\
\hline & & & & & & \\
\hline & & 2. 13.2 & 2. 3.4 & & & \\
\hline \multirow[t]{9}{*}{ Scott (2004) [96] } & \multirow{9}{*}{$\begin{array}{l}\text { 1. Sexual Self Schema Scale } \\
\text { 2. Sexual desire (subscale, Brief Index of } \\
\text { Sexual Function) } \\
\text { 3. Sexual arousal (subscale, Brief Index of } \\
\text { Sexual Function) }\end{array}$} & Baseline, post-treatment mean & Baseline, post-treatment mean score & \multirow{3}{*}{$\begin{array}{l}\text { Effect size, p- } \\
\text { value of couples } \\
\text { coping vs other } \\
\text { two conditions: }\end{array}$} & \multirow{9}{*}{ Moderate } & \multirow[t]{9}{*}{ - Dropout: $11 \%$} \\
\hline & & $\begin{array}{l}\text { score(SD): } \\
\text { Couples coping }\end{array}$ & $\begin{array}{l}\text { (SD): } \\
\text { Medical information }\end{array}$ & & & \\
\hline & & $1.57 .3(13.5), 62.8(12.2)$ & 1. $55.4(14.3), 55.8(11.0)$ & & & \\
\hline & & 2. $4.2(2.8), 4.0(2.3)$ & 2. $3.1(1.9), 2.4(2.4)$ & \multirow{2}{*}{$\begin{array}{r}\text { 1. } d=0.8 \\
p<0.05\end{array}$} & & \\
\hline & & \multirow[t]{5}{*}{ 3. $2.7(2.5), 2.0(2.0)$} & 3. $1.9(1.4), 1.6(1.6)$ & & & \\
\hline & & & Patient coping & \multirow{4}{*}{$\begin{array}{l}\text { 2. No difference } \\
\text { 3. No difference }\end{array}$} & & \\
\hline & & & 1. $55.7(14.3), 56.0(12.0)$ & & & \\
\hline & & & 2. $3.7(2.6), 2.7(2.9)$ & & & \\
\hline & & & 3. 3.1 (1.6), $2.2(2.3)$ & & & \\
\hline
\end{tabular}


intervention group received individualized plans of education, counseling, pharmacologic and/or behavioral interventions, psychosocial support, and referrals compared to controls who underwent usual care. Sexual function was measured by the CARES Sexual Summary Scale, which is scored from 0 to 4 (higher score indicating more severe problems). The mean score change of the intervention group (0.46, $95 \%$ CI 0.30-0.62) was statistically significantly larger than that of the control group (0.11, $95 \% \mathrm{CI}-0.16$ to 0.38 ), $p=0.03$, but clinical relevance is unclear. Most studies intervening on general psychosocial health, rather than targeting sexual health, did not appear to improve sexual function [55, 58-60]. Researchers who undertook group therapy interventions reported difficulties with attendance and higher dropout rates $[49,51]$. Couple-based therapy incorporated counseling on cancer, sexual health, and communication and consistently improved various aspects of sexual function, most frequently sexual satisfaction $[47,50,61]$.

\section{Discussion}

The majority of BCS experience sexual problems in survivorship, most commonly vaginal and vulvar dryness. Despite the significant population of $\mathrm{BCS}$ and high prevalence of sexual problems, the number of RCTs intervening on sexual health was limited. This review summarized evidence for BCS across all ages, because trials in midlife BCS were few. Results showed significant evidence for regular use of vaginal moisturizers to improve dryness, dyspareunia, and sexual satisfaction. Uncontrolled studies with vaginal estradiol, estriol or testosterone also improved vaginal symptoms, but showed systemic absorption. Educational and counseling interventions, particularly those targeting sexual dysfunction, improved various aspects of sexual health. No consistent improvements in sexual health were observed with physical activity, transdermal testosterone or hot flash interventions. Overall for most included studies, the quality of evidence by GRADE criteria was moderate to low.

Vulvovaginal symptoms occur in 20 to $50 \%$ of healthy women of midlife and older as a result of estrogen deprivation [62]. BCS are at heightened risk of these symptoms because chemotherapy, oophorectomy and/or endocrine therapies further decrease estrogen exposure. The clinical trial data show improvements in vaginal dryness, dyspareunia, sexual satisfaction and frequency, and vaginal $\mathrm{pH}$ with regular use at least 2-3 times weekly of a polycarbophil-based vaginal moisturizer. Compliance for at least twelve weeks is important, because major symptom gains occurred between 1-3 months and recur after stopping use, similar to data in the general population [63]. Vulvovaginal symptom relief from regular use of other moisturizers is likely, and $\mathrm{pH}$ balance in products may be important [23, 29]. Among available vaginal moisturizers, BCS should consider preferentially using products with evidence of efficacy.

Use of minimally absorbed local vaginal estrogens and androgens provide vaginal symptom relief, with local estrogens appearing more effective than non-hormonal moisturizers [24, 64]. Even at low doses, estradiol tablets and creams and compounded testosterone are systemically absorbed [24, 28, 30-33]. Unfortunately, there are no clinical trial data on adverse breast cancer outcomes with extended use. Nor are there studies in BCS that compare 7, 10 and 25 micrograms of vaginal estradiol for symptom control and systemic absorption. Whether risk of breast cancer recurrence or death would be higher in estrogen-responsive tumors is also unknown. As local estrogens and androgens are not FDA-approved for use in BCS, these medications are prescribed offlabel and use requires careful discussion between BCS and their healthcare providers.

There was a lack of evidence to support incorporating systemic interventions or physical therapy into the treatment paradigm for sexual dysfunction. The single trial on transdermal testosterone did not demonstrate greater sexual desire compared to the placebo cream after 1 month of use [34]. These findings stand in contrast to several trials in women without prior breast cancer in which androgen therapy improved sexual desire, potentially because these trials were longer in duration (12-24 weeks) and provided supplemental estrogen [35-40]. Notably, there were no clinical trials on treating sexual dysfunction related to serotonin receptor uptake inhibitors in BCS.

Multiple counseling and educational strategies, particularly those targeting sexual dysfunction, have been shown to improve sexual health in BCS. Marriage and family therapists, sex therapists, sexual counselors or psychologists offer counseling interventions. With the aid of online resources, BCS can look for providers who are appropriately educated, credentialed or have significant prior experience with sexual health after cancer. Excellent online resources are found on sites for the American Association of Sexuality Educators, Counselors, and Therapists, the International Society for the Study of Women's Sexual Health, and the American Cancer Society. A number of investigators have designed educational interventions using printed materials, CDs, and websites for content with healthcare provider or peer support [46, 48, 52-54]. This approach is important to study further, as it has the potential advantage of being delivered remotely to extend access to BCS who do not have specialized care locally.

The strength of this review is the systematic approach to identifying and grading current evidence on sexual health interventions specific to breast cancer survivors. 
This approach enabled us to identify the gaps in data. Several interventions that have shown promise in women without a history of breast cancer have not undergone clinical trials in BCS. These include ospemifene and systemic DHEA for the treatment of vulvovaginal symptoms and flibanserin for the treatment of arousal and sexual interest disorders $[65,66]$. The primary limitation was heterogeneity of interventions and outcome measures that restricted the ability to pool data from studies of limited sample size. A recent systematic review sought to evaluate the psychometric properties of sexual dysfunction screening tools and the extent to which they measure DSM-5 aspects of sexual dysfunction for BCS [67]. The review found 31 different scales measuring sexual function, of which the Arizona Sexual Experience Scale, Female Sexual Function Index, and Sexual Problems Scale were determined to meet criteria for acceptable psychometric properties while incorporating DSM-5 areas of sexual dysfunction. Future studies in BCS should carefully consider these outcome measures in study design.

This review demonstrated that current evidence on interventions for improving sexual interest, orgasm and genitopelvic pain in BCS of midlife is limited in quantity and moderate to low in quality. From these data, we recommend prolonged and regular use of non-hormonal vaginal moisturizers to alleviate vulvar and vaginal dryness symptoms and dyspareunia. We also recommend seeking educational and counseling interventions. A number of online resources on sexual health after breast cancer may be useful for BCS and their providers (Table 7). Because each of these interventions have limited efficacy, clinical trials to test novel interventions such as ospemifene are needed in breast cancer survivors.

\section{Appendix \\ PubMed Search Strategy}

(("Clinical Trial"[Publication Type] OR "Comparative Study"[Publication Type]) OR ("Phase I Clinical Trial" OR "Phase II Clinical Trial" OR "Phase III Clinical Trial" OR "Phase IV Clinical Trial" OR "Controlled Clinical Trial" OR "Multicenter Study" OR "Observational Study" OR "Randomized Controlled Trial" OR "Pragmatic Clinical Trial" OR "Comparative Study")).

AND (("Breast Neoplasms"[Mesh] NOT "Breast Neoplasms, Male"[Mesh]) OR ("Breast cancer" OR "Breast Neoplasms")).

AND (("Sexual Dysfunctions, Psychological/prevention and control"[Mesh] OR "Sexual Dysfunctions, Psychological/rehabilitation"[Mesh] OR "Sexual Dysfunctions, Psychological/therapy"[Mesh] OR "Sexual Dysfunction, Physiological/prevention and control"[Mesh] OR "Sexual Dysfunction, Physiological/rehabilitation"[Mesh] OR "Sexual Dysfunction, Physiological/therapy"[Mesh] OR "Vaginal Creams, Foams, and Jellies"[Mesh] OR "Biofeedback, Psychology"[Mesh] OR "Cognitive Therapy"[Mesh] OR "Psychotherapy"[Mesh] OR "Sex Counseling"[Mesh] OR "Patient Education as Topic"[Mesh] OR "Testosterone/ therapy"[Mesh] OR "Antidepressive Agents"[Mesh]) OR

Table 7 Patient Resources: Companion document for use by women seeking management for female sexual dysfunction

\begin{tabular}{|c|c|c|}
\hline What type of information is here? & What organization provides this resource? & Link to website \\
\hline \multirow{2}{*}{$\begin{array}{l}\text { Fact sheet and video describing sexual } \\
\text { problems and treatment options for } \\
\text { the general population. }\end{array}$} & \multirow[t]{2}{*}{ American Society for Reproductive Medicine } & $\begin{array}{l}\text { http://www.reproductivefacts.org/ } \\
\text { FACTSHEET_Sexual_Dysfunction_and_Infertility/ }\end{array}$ \\
\hline & & $\begin{array}{l}\text { http://www.reproductivefacts.org/awards/ } \\
\text { detail.aspx?id=10701 }\end{array}$ \\
\hline $\begin{array}{l}\text { Fact sheets about cancer, its effect on } \\
\text { sex and sexuality, and treatment } \\
\text { options }\end{array}$ & American Cancer Society & $\begin{array}{l}\text { http://www.cancer.org/treatment/ } \\
\text { treatmentsandsideeffects/physicalsideeffects/ } \\
\text { sexualsideeffectsinwomen/sexualityforthewoman/ } \\
\text { index }\end{array}$ \\
\hline $\begin{array}{l}\text { Sexual function screening guidelines } \\
\text { and treatment options for cancer } \\
\text { survivors }\end{array}$ & National Comprehensive Cancer Network & $\begin{array}{l}\text { http://www.nccn.org/professionals/physician_gls/ } \\
\text { pdf/survivorship.pdf }\end{array}$ \\
\hline $\begin{array}{l}\text { Recorded talk by sexual health } \\
\text { providers on rediscovering intimacy } \\
\text { after cancer treatment }\end{array}$ & $\begin{array}{l}\text { Cancer Care, in collaboration with National Cancer } \\
\text { Institute, Livestrong, LBBC, Intercultural Cancer } \\
\text { Council, National Coalition for Cancer Survivorship }\end{array}$ & $\begin{array}{l}\text { http://www.cancercare.org/connect_workshops/138- } \\
\text { cancer_survivorship_2008-05-13 }\end{array}$ \\
\hline $\begin{array}{l}\text { Information on vaginal dryness } \\
\text { treatment options for the general } \\
\text { population }\end{array}$ & North American Menopause Society & $\begin{array}{l}\text { http://www.menopause.org/docs/for-women/ } \\
\text { mndryness.pdf }\end{array}$ \\
\hline $\begin{array}{l}\text { Online forum to talk to other breast } \\
\text { cancer survivors about sexual concerns }\end{array}$ & BreastCancer.org & http://www.breastcancer.org/tips/intimacy \\
\hline Finding a sexual health provider & $\begin{array}{l}\text { American Association of Sexuality Educations, } \\
\text { Counselors, and Therapists }\end{array}$ & http://www.aasect.org/referral-directory \\
\hline Finding a sexual health provider & Society for Sex Therapy and Research & http://www.sstarnet.org/therapist-directory.php \\
\hline
\end{tabular}


("vaginal lubricant" OR "vaginal moisturizer" OR "pelvic floor muscle relaxation" OR "pelvic floor physical therapy" OR "pelvic floor muscle training" OR "biofeedback" OR "vaginal dilator" OR "biofeedback" OR "cognitive behavioral therapy" OR ("sex" AND "therapy") OR "psychotherapy" OR "sex counseling" OR "patient education" OR "testosterone" OR "antidepressant" OR "treatment" OR "flibanserin" OR "ospemifene" OR "vibrator" OR "vaginal dilator" OR "DHEA")).

AND (("Sexual Dysfunction, Physiological"[Mesh] OR "Interpersonal Relations"[Mesh] OR "Sexual Behavior"[Mesh] OR "Vaginismus"[Mesh] OR "Coitus"[Mesh] OR "Libido"[Mesh] OR "Orgasm"[Mesh]) OR ("dyspareunia" OR "coitus" OR "coital frequency" OR "intercourse frequency" OR "vaginal dryness" OR "sexual lubrication" OR "vulvovaginal atrophy" OR "libido" OR "sexual function" OR "sexual dysfunction" OR "sexual interest" OR "sexual desire" OR "sexual arousal" OR "orgasm" OR "sexual pleasure" OR "sexual dissatisfaction" OR "sex" OR "intimacy" OR "sexual desire disorder" OR "sexual arousal disorder" OR "orgasmic disorder" OR "sexual pain disorder")).

\section{Competing interests}

The authors declare that they have no competing interests.

\section{Authors' contributions}

SS, SD, BS and IS conducted the initial search and screen for inclusion papers. IS and SS drafted the Summary of Studies and Outcomes Tables. IS and SD assessed risk of bias for each study. All authors were involved in drafting the manuscript or revising it critically for intellectual accuracy. All authors read and approved the final manuscript.

\section{Acknowledgements}

This study was financially supported by the Breast Cancer Research Program 120500-PFT-11-008-01-CPPB, HD-058799-01. The funding organization was not involved in the study design, data collection, data analyses, or writing of the manuscript for publication.

\section{Author details}

'Department of Reproductive Medicine and Moores Cancer Center, University of California, San Diego, 3855 Health Sciences Dive \#0901, La Jolla, CA 92093, USA. ${ }^{2}$ Young Survival Coalition, 80 Broad Street, New York, NY 10004, USA. ${ }^{3}$ University of California, Irvine, Beckman Laser Institute, 1002 Health Sciences Road, Irvine, CA 92612, USA. ${ }^{4}$ Southern California Center for Sexual Health and Survivorship Medicine, 1501 Superior Avenue, Newport Beach, CA 92663, USA.

Received: 12 May 2015 Accepted: 2 September 2015

Published online: 02 November 2015

\section{References}

1. American Cancer Society. Cancer Treatment and Survivorship Facts \& Figs. 2014-2015. Atlanta: American Cancer Society; 2014.

2. Baron RH, Kelvin JF, Bookbinder M, Cramer L, Borgen PI, Thaler HT. Patients' sensations after breast cancer surgery. A pilot study. Cancer Pract. 2000:8(5):215-22.

3. Bines J, Oleske DM, Cobleigh MA. Ovarian function in premenopausal women treated with adjuvant chemotherapy for breast cancer. J Clin Oncol. 1996;14(5):1718-29.

4. Onen Sertoz O, Elbi Mete H, Noyan A, Alper M, Kapkac M. Effects of surgery type on body image, sexuality, self-esteem, and marital adjustment in breast cancer: a controlled study. Turkish J Psychiatry. 2004;15(4):264-75.

5. Pelusi J. Sexuality and body image. Cancer Nurs. 2006;29(2 Suppl):32-8.

6. Wilmoth MC, Ross JA. Women's perception. Breast cancer treatment and sexuality. Cancer Pract. 1997;5(6):353-9.
7. Hawkins Y, Ussher J, Gilbert E, Perz J, Sandoval M, Sundquist K. Changes in sexuality and intimacy after the diagnosis and treatment of cancer: the experience of partners in a sexual relationship with a person with cancer. Cancer Nurs. 2009;32(4):271-80. doi:10.1097/NCC.0b013e31819b5a93.

8. Ganz PA, Rowland JH, Meyerowitz BE, Desmond KA. Impact of different adjuvant therapy strategies on quality of life in breast cancer survivors. Recent Results Cancer Res. 1998;152:396-411.

9. Ganz PA, Rowland JH, Desmond K, Meyerowitz BE, Wyatt GE. Life after breast cancer: understanding women's health-related quality of life and sexual functioning. J Clin Oncol. 1998;16(2):501-14.

10. American Psychiatric Association. Diagnostic and statistical manual of mental disorders. 5th ed. Arlington: American Psychiatric Association; 2013.

11. Fobair P, Stewart SL, Chang S, D'Onofrio C, Banks PJ, Bloom JR. Body image and sexual problems in young women with breast cancer. Psychooncology. 2006;15(7):579-94. doi:10.1002/pon.991.

12. Bloom JR, Stewart SL, Oakley-Girvan I, Banks PJ, Shema S. Quality of life of younger breast cancer survivors: persistence of problems and sense of wellbeing. Psycho-oncology. 2012;21(6):655-65. doi:10.1002/pon.1965.

13. Burwell SR, Case LD, Kaelin C, Avis NE. Sexual problems in younger women after breast cancer surgery. J Clin Oncol. 2006;24(18):2815-21. doi:10.1200/ jco.2005.04.2499.

14. Avis NE, Crawford S, Manuel J. Quality of life among younger women with breast cancer. J Clin Oncol. 2005:23(15):3322-30. doi:10.1200/jco.2005.05.130.

15. Kornblith AB, Powell M, Regan MM, Bennett S, Krasner C, Moy B, et al. Long-term psychosocial adjustment of older vs younger survivors of breast and endometrial cancer. Psycho-oncology. 2007;16(10):895-903. doi:10.1002/pon.1146.

16. Flynn KE, Reese JB, Jeffery DD, Abernethy AP, Lin L, Shelby RA, et al. Patient experiences with communication about sex during and after treatment for cancer. Psycho-oncology. 2012;21(6):594-601. doi:10.1002/pon.1947.

17. Park ER, Bober SL, Campbell EG, Recklitis CJ, Kutner JS, Diller L. General internist communication about sexual function with cancer survivors. J Gen Intern Med. 2009;24 Suppl 2:S407-11. doi:10.1007/s11606-009-1026-5.

18. Ussher JM, Perz J, Gilbert E, Wong WK, Mason C, Hobbs K, et al. Talking about sex after cancer: a discourse analytic study of health care professional accounts of sexual communication with patients. Psychol Health. 2013;28(12):1370-90. doi:10.1080/08870446.2013.811242.

19. Bachmann GA, Leiblum SR, Grill J. Brief sexual inquiry in gynecologic practice. Obstet Gynecol. 1989;73(3 Pt 1):425-7.

20. Mao JJ, Bowman MA, Stricker CT, DeMichele A, Jacobs L, Chan D, et al. Delivery of survivorship care by primary care physicians: the perspective of breast cancer patients. J Clin Oncol. 2009;27(6):933-8. doi:10.1200/jco.2008.18.0679.

21. Moher D, Liberati A, Tetzlaff J, Altman DG. Preferred reporting items for systematic reviews and meta-analyses: the PRISMA statement. PLOS Med. 2009;6(7), e1000097. doi:10.1371/journal.pmed.1000097.

22. Higgins JP, Altman DG, Gotzsche PC, Juni P, Moher D, Oxman AD, et al. The Cochrane Collaboration's tool for assessing risk of bias in randomised trials. BMJ. 2011;343:d5928. doi:10.1136/bmj.d5928.

23. Loprinzi CL, Abu-Ghazaleh S, Sloan JA, Van Haelst-Pisani C, Hammer AM, Rowland Jr KM, et al. Phase III randomized double-blind study to evaluate the efficacy of a polycarbophil-based vaginal moisturizer in women with breast cancer. J Clin Oncol. 1997;15(3):969-73.

24. Biglia N, Peano E, Sgandurra P, Moggio G, Panuccio E, Migliardi M, et al. Low-dose vaginal estrogens or vaginal moisturizer in breast cancer survivors with urogenital atrophy: a preliminary study. Gynecol Endocrinol. 2010;26(6):404-12. doi:10.3109/09513591003632258

25. Gelfand MM, Wendman E. Treating vaginal dryness in breast cancer patients: results of applying a polycarbophil moisturizing gel. J Women's Health. 1994:3(6):427-34.

26. Juraskova I, Jarvis S, Mok K, Peate M, Meiser B, Cheah BC, et al. The acceptability, feasibility, and efficacy (phase I/II study) of the OVERcome (Olive Oil, Vaginal Exercise, and MoisturizeR) intervention to improve dyspareunia and alleviate sexual problems in women with breast cancer. J Sex Med. 2013;10(10):2549-58. doi:10.1111/jsm.12156.

27. Dahir M, Travers-Gustafson D. Breast cancer, aromatase inhibitor therapy, and sexual functioning: a pilot study of the effects of vaginal testosterone therapy. Sexual Med. 2014;2(1):8-15. doi:10.1002/sm2.22.

28. Witherby S, Johnson J, Demers L, Mount S, Littenberg B, Maclean CD, et al. Topical testosterone for breast cancer patients with vaginal atrophy related to aromatase inhibitors: a phase I/II study. The Oncologist. 2011;16(4):424-31. doi:10.1634/theoncologist.2010-0435. 
29. Lee YK, Chung HH, Kim JW, Park NH, Song YS, Kang SB. Vaginal pHbalanced gel for the control of atrophic vaginitis among breast cancer survivors: a randomized controlled trial. Obstet Gynecol. 2011;117(4):922-7. doi:10.1097/AOG.0b013e3182118790.

30. Pfeiler G, Glatz C, Konigsberg R, Geisendorfer T, Fink-Retter A, Kubista E, et al. Vaginal estriol to overcome side-effects of aromatase inhibitors in breast cancer patients. Climacteric. 2011;14(3):339-44. doi:10.3109/13697137.2010.529967.

31. Kendall A, Dowsett M, Folkerd E, Smith I. Caution: Vaginal estradiol appears to be contraindicated in postmenopausal women on adjuvant aromatase inhibitors. Ann Oncol. 2006;17(4):584-7. doi:10.1093/annonc/mdj127.

32. Donders $G$, Neven P, Moegele M, Lintermans A, Bellen G, Prasauskas V, et al. Ultra-low-dose estriol and Lactobacillus acidophilus vaginal tablets (Gynoflor((R))) for vaginal atrophy in postmenopausal breast cancer patients on aromatase inhibitors: pharmacokinetic, safety, and efficacy phase I clinical study. Breast Cancer Res Treat. 2014;145(2):371-9. doi:10.1007/s10549-014-2930-x.

33. Wills S, Ravipati A, Venuturumilli P, Kresge C, Folkerd E, Dowsett M, et al. Effects of vaginal estrogens on serum estradiol levels in postmenopausa breast cancer survivors and women at risk of breast cancer taking an aromatase inhibitor or a selective estrogen receptor modulator. J Oncol Pract. 2012;8(3):144-8. doi:10.1200/jop.2011.000352.

34. Barton DL, Wender DB, Sloan JA, Dalton RJ, Balcueva EP, Atherton PJ, et al. Randomized controlled trial to evaluate transdermal testosterone in female cancer survivors with decreased libido; North Central Cancer Treatment Group protocol N02C3. J Natl Cancer Inst. 2007;99(9):672-9. doi:10.1093/jnci/djk149.

35. Shifren JL, Braunstein GD, Simon JA, Casson PR, Buster JE, Redmond GP, et al. Transdermal testosterone treatment in women with impaired sexual function after oophorectomy. N Engl J Med. 2000;343(10):682-8. doi:10.1056/nejm200009073431002.

36. Goldstat R, Briganti E, Tran J, Wolfe R, Davis SR. Transdermal testosterone therapy improves well-being, mood, and sexual function in premenopausal women. Menopause. 2003;10(5):390-8. doi:10.1097/01.gme.0000060256.03945.20.

37. Braunstein GD, Sundwall DA, Katz M, Shifren JL, Buster JE, Simon JA, et al. Safety and efficacy of a testosterone patch for the treatment of hypoactive sexual desire disorder in surgically menopausal women: a randomized, placebo-controlled trial. Arch Intern Med. 2005;165(14):1582-9. doi:10.1001/ archinte.165.14.1582.

38. Buster JE, Kingsberg SA, Aguirre O, Brown C, Breaux JG, Buch A, et al. Testosterone patch for low sexual desire in surgically menopausal women: a randomized trial. Obstet Gynecol. 2005;105(5 Pt 1):944-52. doi:10.1097/ 01.AOG.0000158103.27672.0d

39. Simon J, Braunstein G, Nachtigall L, Utian W, Katz M, Miller S, et al. Testosterone patch increases sexual activity and desire in surgically menopausal women with hypoactive sexual desire disorder. J Clin Endocrinol Metab. 2005:90(9):5226-33. doi:10.1210/jc.2004-1747.

40. Nathorst-Boos J, Floter A, Jarkander-Rolff M, Carlstrom K, Schoultz B. Treatment with percutanous testosterone gel in postmenopausal women with decreased libido-effects on sexuality and psychological general wellbeing. Maturitas. 2006;53(1):11-8. doi:10.1016/.maturitas.2005.01.002.

41. Nunez GR, Pinczowski H, Zanellato R, Tateyama L, Schindler F, Fonseca F, et al. Bupropion for control of hot flashes in breast cancer survivors: a prospective, double-blind, randomized, crossover, pilot phase II trial. Journal of Pain and Symptom Management. 2013;45(6):969-79. doi:10.1016/ j.jpainsymman.2012.06.011

42. Buijs $\mathrm{C}$, Mom CH, Willemse PH, Marike Boezen H, Maurer JM, Wymenga AN, et al. Venlafaxine versus clonidine for the treatment of hot flashes in breast cancer patients: a double-blind, randomized cross-over study. Breast Cancer Res Treat. 2009;115(3):573-80. doi:10.1007/s10549-008-0138-7.

43. Duijts SFA, Stolk-Vos AC, Oldenburg HSA, Van Beurden M, Aaronson NK. Characteristics of breast cancer patients who experience menopausal transition due to treatment. Climacteric. 2011;14(3):362-8. doi:10.3109/13697137.2011.557163.

44. Speck RM, Gross CR, Hormes JM, Ahmed RL, Lytle LA, Hwang WT, et al. Changes in the Body Image and Relationship Scale following a one-year strength training trial for breast cancer survivors with or at risk for lymphedema. Breast Cancer Res Treat. 2010;121(2):421-30. doi:10.1007/ s10549-009-0550-7.

45. Berglund G, Bolund C, Gustafsson U, Sjoden P. A randomized study of a rehabilitation program for cancer patients: the 'starting again' group. Psycho-oncology. 1994;3:109-20.

46. Anderson DJ, Seib C, McCarthy AL, Yates P, Porter-Steele J, McGuire A, et al. Facilitating lifestyle changes to manage menopausal symptoms in women with breast cancer: a randomized controlled pilot trial of The Pink Women's Wellness Program. Menopause. 2015. doi:10.1097/ gme.0000000000000421

47. Baucom DH, Porter LS, Kirby JS, Gremore TM, Wiesenthal N, Aldridge W, et al. A couple-based intervention for female breast cancer. Psychooncology. 2009;18(3):276-83. doi:10.1002/pon.1395.

48. Ganz PA, Greendale GA, Petersen L, Zibecchi L, Kahn B, Belin TR. Managing menopausal symptoms in breast cancer survivors: results of a randomized controlled trial. J Natl Cancer Inst. 2000;92(13):1054-64.

49. Jun EY, Kim S, Chang SB, Oh K, Kang HS, Kang SS. The effect of a sexual life reframing program on marital intimacy, body image, and sexual function among breast cancer survivors. Cancer Nurs. 2011;34(2):142-9. doi:10.1097/ NCC.0b013e3181f1ab7a.

50. Kalaitzi C, Papadopoulos VP, Michas K, Vlasis K, Skandalakis P, Filippou D. Combined brief psychosexual intervention after mastectomy: effects on sexuality, body image, and psychological well-being. J Surg Oncol. 2007;96(3):235-40. doi:10.1002/jso.20811.

51. Rowland JH, Meyerowitz BE, Crespi CM, Leedham B, Desmond K, Belin TR, et al. Addressing intimacy and partner communication after breast cancer: a randomized controlled group intervention. Breast Cancer Res Treat. 2009:118(1):99-111. doi:10.1007/s10549-009-0398-x.

52. Schover LR, Jenkins R, Sui D, Adams JH, Marion MS, Jackson KE. Randomized trial of peer counseling on reproductive health in African American breast cancer survivors. J Clin Oncol. 2006;24(10):1620-6. doi:10.1200/jco.2005.04.7159.

53. Schover $L R$, Rhodes MM, Baum G, Adams JH, Jenkins $R$, Lewis $P$, et al. Sisters Peer Counseling in Reproductive Issues After Treatment (SPIRIT): a peer counseling program to improve reproductive health among African American breast cancer survivors. Cancer. 2011;117(21):4983-92. doi:10.1002/cncr.26139.

54. Schover LR, Yuan Y, Fellman BM, Odensky E, Lewis PE, Martinetti P. Efficacy trial of an Internet-based intervention for cancer-related female sexual dysfunction. J Natl Compr Cancer Netw. 2013;11(11):1389-97.

55. Allen SM, Shah AC, Nezu AM, Nezu CM, Ciambrone D, Hogan J, et al. A problem-solving approach to stress reduction among younger women with breast carcinoma: a randomized controlled trial. Cancer. 2002;94(12):3089-100. doi:10.1002/cncr.10586.

56. Germino B, Mishel M, Crandell J, Porter L, Blyler D, Jenerette $C$, et al. Outcomes of an uncertainty management intervention in younger African American and Caucasian breast cancer survivors. Oncol Nurs Forum. 2013:40(1):82-92. doi:10.1188/13.ONF.82-92.

57. Marcus AC, Garrett KM, Cella D, Wenzel L, Brady MJ, Fairclough D, et al. Can telephone counseling post-treatment improve psychosocial outcomes among early stage breast cancer survivors? Psycho-oncology. 2010;19(9):923-32. doi:10.1002/pon.1653.

58. Salonen P, Tarkka MT, Kellokumpu-Lehtinen PL, Koivisto AM, Aalto P, Kaunonen M. Effect of social support on changes in quality of life in early breast cancer patients: a longitudinal study. Scandinavian Journal of Caring Sciences. 2013;27(2):396-405. doi:10.1111/j.1471-6712.2012.01050.x.

59. Greer S, Moorey S, Baruch JD, Watson M, Robertson BM, Mason A, et al. Adjuvant psychological therapy for patients with cancer: a prospective randomised trial. BMJ. 1992;304(6828):675-80.

60. Vos PJ, Garssen B, Visser AP, Duivenvoorden HJ, de Haes HC. Psychosocial intervention for women with primary, non-metastatic breast cancer: a comparison between participants and non-participants. Psychother Psychosom. 2004;73(5):276-85. doi:10.1159/000078844.

61. Christensen D. Postmastectomy couple counseling: an outcome study of a structured treatment protocol. J Sex Marital Therapy. 1983;9(4):266-75. doi:10.1080/00926238308410913.

62. North Amer Menopause S. Management of symptomatic vulvovaginal atrophy: 2013 position statement of The North American Menopause Society. Menopause. 2013;20(9):888-902. doi:10.1097/gme.0b013e3182a122c2.

63. Bygdeman M, Swahn ML. Replens versus dienoestrol cream in the symptomatic treatment of vaginal atrophy in postmenopausal women. Maturitas. 1996;23(3):259-63.

64. Rahn DD, Carberry C, Sanses TV, Mamik MM, Ward RM, Meriwether KV, et al. Vaginal estrogen for genitourinary syndrome of menopause: a systematic review. Obstet Gynecol. 2014;124(6):1147-56. doi:10.1097/ aog.0000000000000526.

65. Portman DJ, Bachmann GA, Simon JA. Ospemifene, a novel selective estrogen receptor modulator for treating dyspareunia associated with postmenopausal vulvar and vaginal atrophy. Menopause. 2013;20(6):623-30. doi:10.1097/gme.0b013e318279ba64. 
66. Tan O, Bradshaw K, Carr BR. Management of vulvovaginal atrophy-related sexual dysfunction in postmenopausal women: an up-to-date review. Menopause. 2012;19(1):109-17. doi:10.1097/gme.0b013e31821f92df.

67. Bartula I, Sherman KA. Screening for sexual dysfunction in women diagnosed with breast cancer: systematic review and recommendations. Breast Cancer Res Treat. 2013;141(2):173-85. doi:10.1007/s10549-013-2685-9.

68. McGahuey CA, Gelenberg AJ, Laukes CA, Moreno FA, Delgado PL, McKnight KM, et al. The Arizona Sexual Experience Scale (ASEX): reliability and validity. J Sex Marital Ther. 2000;26(1):25-40.

69. Mathias C, Athanazio RA, Braghiroli Ml, et al. Use of the Arizona Sexual Experience Scale (ASEX) in the evaluation of sexual dysfunction in Brazilian cancer patients. [in Spanish]. J Bras Psiquiatr. 2005;54:216-20.

70. Hormes JM, Lytle LA, Gross CR, Ahmed RL, Troxel AB, Schmitz KH. The body image and relationships scale: development and validation of a measure of body image in female breast cancer survivors. J Clin Oncol. 2008;26(8):1269-74. doi:10.1200/jco.2007.14.2661.

71. Schag CAC, Heinrich RL. Cancer Rehabilitation Evaluation System (CARES) manual. CARES Consultants: Santa Monica; 1988.

72. Clayton AH, McGarvey EL, Clavet GJ. The Changes in Sexual Functioning Questionnaire (CSFQ): development, reliability, and validity. Psychopharmacol Bull. 1997;33(4):731-45.

73. Derogatis LR. The Derogatis Interview for Sexual Functioning (DISF/DISF-SR): an introductory report. J Sex Marital Ther. 1997;23(4):291-304. doi:10.1080/ 00926239708403933

74. Sprangers MA, Groenvold M, Arraras JI, Franklin J, te Velde A, Muller, M et al. The European Organization for Research and Treatment of Cancer breast cancer-specific quality-of-life questionnaire module: first results from a three-country field study. J Clin Oncol. 1996;14(10):2756-68.

75. Wiegel M, Meston C, Rosen R. The female sexual function index (FSFI): crossvalidation and development of clinical cutoff scores. J Sex Marital Ther. 2005;31(1):1-20. doi:10.1080/00926230590475206.

76. Fallowfield L, Leaity SK, Howell A, Benson S, Cella D. Assessment of quality of life in women undergoing hormonal therapy for breast cancer: validation of an endocrine symptom subscale for the FACT-B. Breast Cancer Res Treat. 1999;55(2):189-99.

77. Waring EM, Reddon JR. The measurement of intimacy in marriage: the Waring Intimacy Questionnaire. J Clin Psychol. 1983;39(1):53-7.

78. Ware Jr JE, The SCD, MOS. 36-item short-form health survey (SF-36). I. Conceptual framework and item selection. Med Care. 1992;30(6):473-83.

79. Rosen RC, Lobo RA, Block BA, Yang HM, Zipfel LM. Menopausal Sexual Interest Questionnaire (MSIQ): a unidimensional scale for the assessment of sexual interest in postmenopausal women. J Sex Marital Ther. 2004;30(4):235-50. doi:10.1080/00926230490422340.

80. Ganz PA, Day R, Ware Jr JE, Redmond C, Fisher B. Base-line quality-of-life assessment in the National Surgical Adjuvant Breast and Bowel Project Breast Cancer Prevention Trial. J Natl Cancer Inst. 1995;87(18):1372-82.

81. McHorney CA, Rust J, Golombok S, Davis S, Bouchard C, Brown C, et al. Profile of Female Sexual Function: a patient-based, international, psychometric instrument for the assessment of hypoactive sexual desire in oophorectomized women. Menopause. 2004;11(4):474-83.

82. Derogatis LR. The psychosocial adjustment to illness scale (PAIS) J Psychosom Res. 1986;30(1):77-91.

83. Norton R. Measuring marital quality: a critical look at the dependent variable. J Marriage Fam. 1983;45:141-51.

84. Thirlaway K, Fallowfield L, Cuzick J. The Sexual Activity Questionnaire: a measure of women's sexual functioning. Quality Life Res. 1996;5(1):81-90.

85. Taylor JF, Rosen RC, Leiblum SR. Self-report assessment of female sexual function: psychometric evaluation of the brief index of sexual functioning for women. Arch Sex Behav. 1994;23(6):627-643.

86. Greene JG. Constructing a standard climacteric scale. Maturitas. 1998;29(1):25-31.

87. Crane R. Sexual satisfaction scale. 1977.

88. Kim SNCSB, Kang HS. Development of sexual satisfaction measurement tool. J Korean Acad Nurs. 1997;27(4):753-64.

89. Andersen BL, Cyranowski JM. Women's sexual self schema. J Pers Soc Psychol. 1994;67:1079-100

90. Speroff L. Efficacy and tolerability of a novel estradiol vaginal ring for relief of menopausal symptoms. Obstet Gynecol. 2003;102(4):823-34.

91. Davila GW, Singh A, Karapanagiotou I, Woodhouse S, Huber K, Zimberg S, et al. Are women with urogenital atrophy symptomatic? Am J Obstet Gynecol. 2003;188(2):382-8.
92. Rioux JE, Devlin C, Gelfand MM, Steinberg WM, Hepburn DS. 17betaestradiol vaginal tablet versus conjugated equine estrogen vaginal cream to relieve menopausal atrophic vaginitis. Menopause. 2000;7(3):156-61.

93. Bachmann G. Urogenital ageing: an old problem newly recognized. Maturitas. 1995;22(Suppl):S1-5.

94. Bachmann GA, Notelovitz M, Gonzalez SJ, Thompson C, Morecraft BA. Vaginal dryness in menopausal women: clinical characteristics and nonhormonal treatment. Clinical Practice Sexuaity. 1991;7(9):1-8.

95. Hustin J, Van den Eynde JP. Cytologic evaluation of the effect of various estrogens given in postmenopause. Acta Cytol. 1977;21(2):225-8.

96. Scott JL, Halford WK, Ward, BG. United we stand? The effects of a couplecoping intervention on adjustment to early stage breast or gynecologicalcancer. J Consult Clin Psychol. 2004;72(6):1122-35.

\section{Submit your next manuscript to BioMed Central and take full advantage of:}

- Convenient online submission

- Thorough peer review

- No space constraints or color figure charges

- Immediate publication on acceptance

- Inclusion in PubMed, CAS, Scopus and Google Scholar

- Research which is freely available for redistribution

Submit your manuscript at www.biomedcentral.com/submit 